\section{OAK RIDGE NATIONAL LABORATORY}

MARTIN MARIETEAA
Conceptual Strategy for Design, Implementation, and Validation of a Biomarker-Based Biomonitoring Capability

\author{
J. F. McCarthy \\ R. S. Halbrook \\ L. R. Shugart
}

Environmental Sciences Division

Publication No. 3702 
This report has been reproduced directly from the best available copy.

Available to DOE and DOE contractors from the office of scientific and Technical Information, P. O. Box 62, Oak Ridge, TN 37831; prices available from (615) 576-8401, FTS 626-8401.

Available to the public from the National Technical Information Service, U.S. Department of Commerce, 5285 Port Royal kd., Springfield, VA 22161 . NTIS price codes-Printed Copy: A06 Microfiche A01

This report was prepared as an account of work sponsored by an agency of the United States Government. Neither the United States Government nor any agency thereof, rier any of their employees, makes any warranty, express or implied, or assumes any legal liability or responsibility for the accuracy, completeness, or usefulness of any information, apparatus, product, or process disclosed, or represents that its u,e would not infringe privately owned rights. Reference herein to any specific conmercial product, process, or service by trade name, trademark, manufacturer, or otherwise, does not necessarily constitute or imply its endorsement, recormenda".ion, or favoring by the United States Government or any agency thereof. The views and opinions of authors expressed herein do not necessarily state or reflect those of the United States Government or any agency thereof. 


\title{
CONCEPTUAL STRATEGY FOR DESIGN, IMPLEMENTATION, AND VALIDATION OF A BIOMARKER-BASED BIOMONITORING CAPABILITY
}

\author{
John F. McCarthy, Richard S. Halbrook, and Lee R. Shugart \\ Environmental Sciences Division \\ Publication No. 3702
}

Date Published: December 1991

Part of the work described in this report has been supported by the U.S. Environmental Protection Agency; however, it has not been subjected to Agency review, therefore it does not necessarily reflect the views of the Agency, and no official endorsement should be inferred.

Prepared by the

OAK RIDGE NATIONAL LABORATORY

Oak Ridge, Tennessee 37831-6285

MASTER

MARTIN MARIETTA ENERGY SYSTEMS, INC.

for the

U. S. DEPARTMENT OH ENERGY

under Contract DE-AC05-84OR21400 


\section{CONTENTS}

FIGURES $\ldots \ldots \ldots \ldots \ldots \ldots \ldots \ldots \ldots \ldots \ldots \ldots \ldots \ldots$ iv

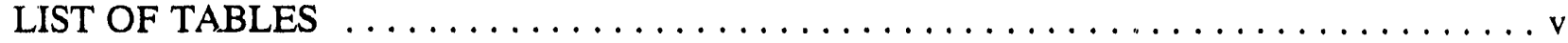

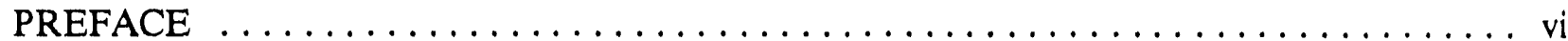

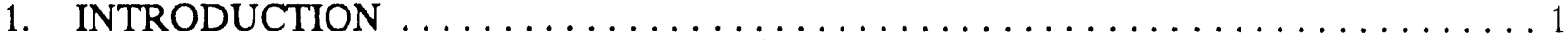

1.1 BIOLOGICAL MARKERS OF ENVIRONMENTAL CONTAMINATION . . . . . . 2

1.2 RATIONALE AND APPLICATION OF BIOMARKER INDICATORS $\ldots \ldots \ldots 4$

1.2.1 Limitations of Existing Approaches to Environmental Assessment . . . . . . . . 5

1.2.2 Biomarkers as Sensitive Indicators of Response to

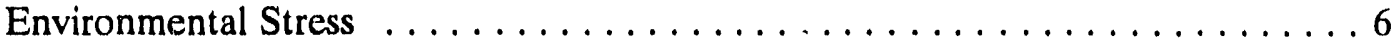

1.2.3 Linking Toxic Exposure to Effects $\ldots \ldots \ldots \ldots \ldots \ldots \ldots \ldots \ldots$

1.2.4 General Indicators vs Chemical-Specific Indicators $\ldots \ldots \ldots \ldots \ldots \ldots \ldots$

1.3 CONSIDERATIONS FOR DESIGNING AND INTERPRETING BIOMARKER

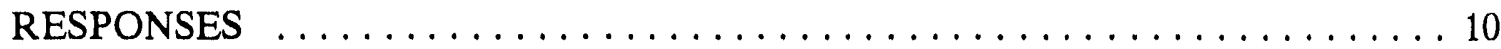

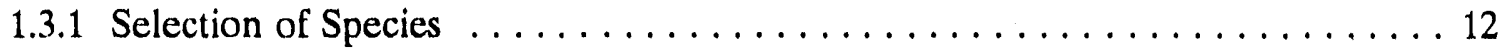

1.3.2 Effects of Physiological and Environmental Variables $\ldots \ldots \ldots \ldots \ldots \ldots \ldots 13$

1.4 GENERAL LIMITATIONS OF BIOMARKERS $\ldots \ldots \ldots \ldots \ldots \ldots \ldots \ldots \ldots \ldots$

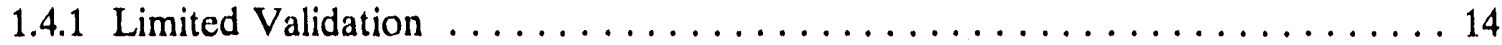

1.4.2 Experience Not Uniform Across Ecosystem Types . . . . . . . . . . . . 14

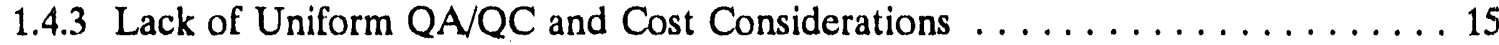

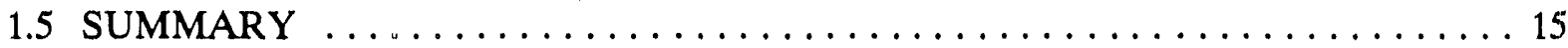

2. CONCEPTUAL PARADIGMS FOR EVALUATING BIOMARKER RESPONSE $\ldots 17$

3. STATISTICAL EVALUATION AND INTERPRETATION OF BIOMARKER

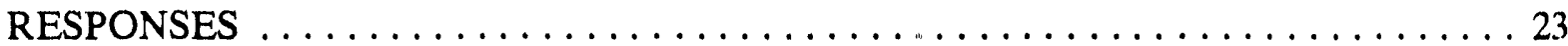

3.1 DIFFICULTIES IN EVALUATING BIOMARKER RESPONSES $\ldots \ldots \ldots \ldots \ldots 23$

3.2 ANALYSIS AND INTERPRETATION OF BIOMARKER RESPONSES $\ldots \ldots \ldots 23$

4. DEFINITION OF APPLICATIONS AND OBJECTIVES OF BIOMARKER-BASED

BIOMONITORING . . . . . . . . . . . . . . . . . . . . . . . . . . . . 29

5. CONCEPTUAL STRATEGY FOR DEVELOPMENT AND APPLICATION OF A

BIOMARKER-BASED BIOMONITORING PROGRAM $\ldots \ldots \ldots \ldots \ldots \ldots \ldots \ldots$

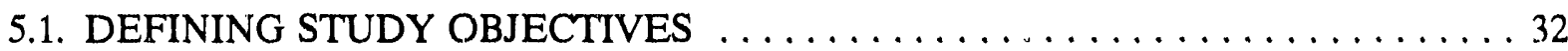

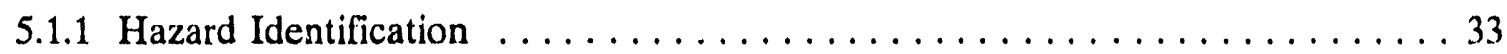

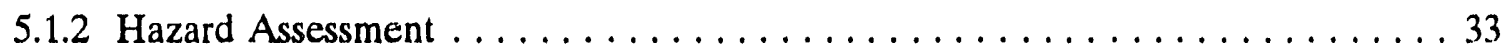

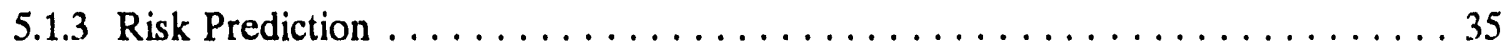

5.2. GENERAL ELEMENTS OF A BIOMARKER-BASED BIOMONITORING

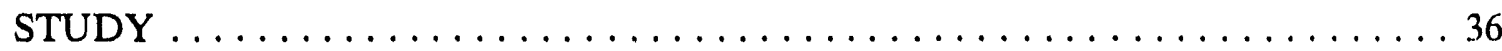

5.2.1 Defining Study Area and Selection of Reference Sites $\ldots \ldots \ldots \ldots \ldots 38$

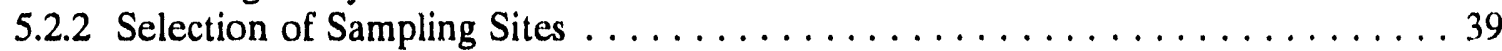

5.2 .3 Characterization of Study and Reference Sites $\ldots \ldots \ldots \ldots \ldots \ldots \ldots$. . . . . . . .

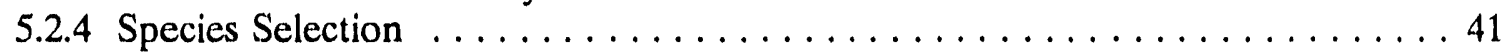

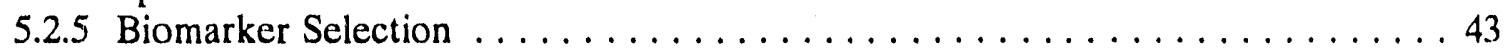

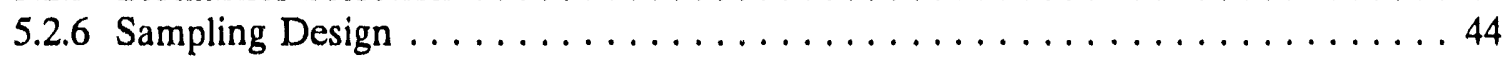

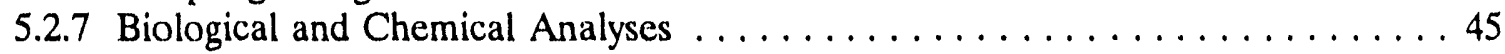

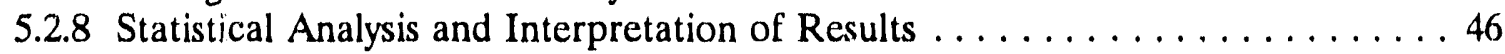

5.3. TAILORING STUDY DESIGN FOR SPECIFIC OBJECTIVES $\ldots \ldots \ldots \ldots \ldots$ 
5.4. RESEARCH COMPONENTS IN DEVELOPMENT OF BIOMARKERS:

LABORATORY AND FIELD STUDIES $\ldots \ldots \ldots \ldots \ldots \ldots \ldots \ldots \ldots \ldots$

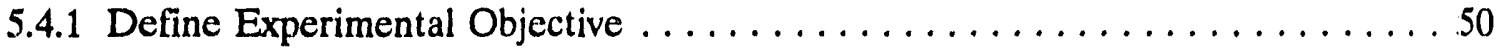

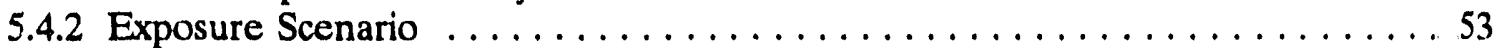

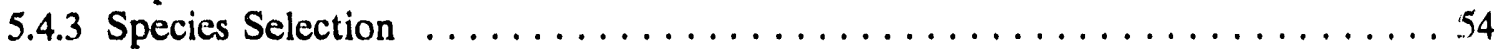

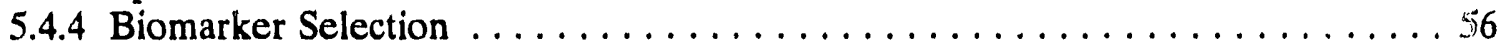

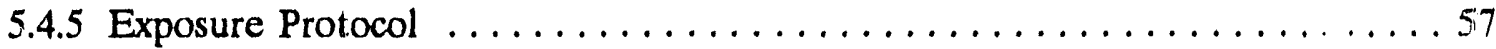

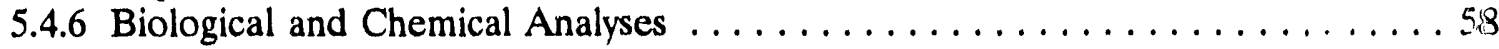

5.4.7 Statistical Analysis and Interpretation of Results . . . . . . . . . . . . 59)

5.4 .8 Comparison of Field and Laboratory Results . . . . . . . . . . . . . 6()

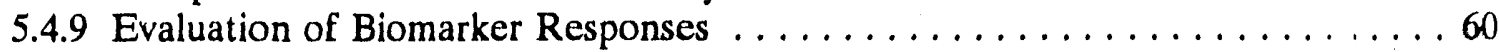

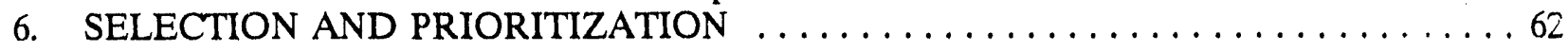

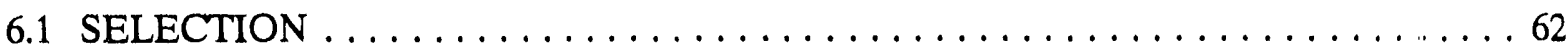

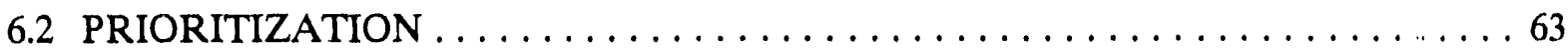

7. BIOMARKERS AS ENDPOINTS IN TOXICITY TESTING $\ldots \ldots \ldots \ldots \ldots \ldots$

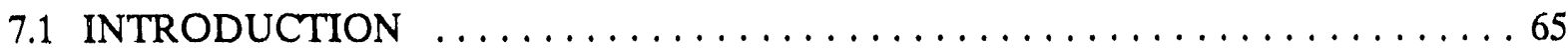

7.2 CASE-IN-POINT: GENOTOXICITY TESTING IN FISH $\ldots \ldots \ldots \ldots \ldots \ldots$

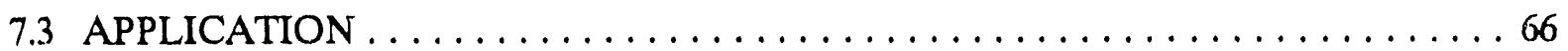

8. LONG-TERM STRATEGY FOR DEVELOPING AND IMPLEMENTING A

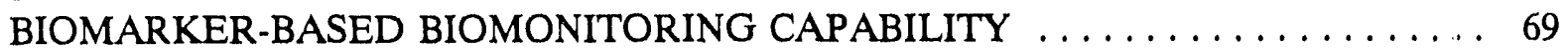

8.1 STATUS OF CURRENT CAPABILITIES $\ldots \ldots \ldots \ldots \ldots \ldots \ldots \ldots \ldots \ldots$

8.2 PROPOSED IMPLEMENTATION OF BIOMARKER MONITORING PROGRAM 70

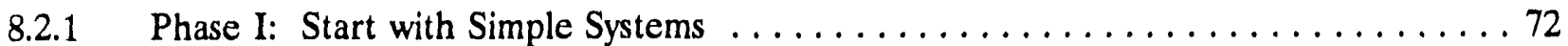

8.2.2 Phase II: Gradually Evolve to More Complex Systems . . . . . . . . . . 73

8.2.3 Phase III: Linking Biomarker Responses to

Community-Level Effects . . . . . . . . . . . . . . . . . . . . 74

8.2.4 Laboratory-Field Interaction: Development, Standardization, and Validation of Key Biomarkers . . . . . . . . . . . . . . 77

8.2.5 Phase IV: Linking Biomarker Responses in Sentinel Species to

Human Epidemiology . . . . . . . . . . . . . . . . . . 78

8.3 SCHEDULE FOR IMPLEMENTATION OF BIOMARKER MONITORING

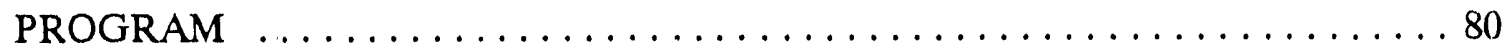

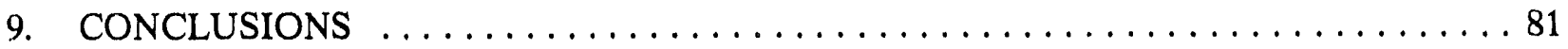

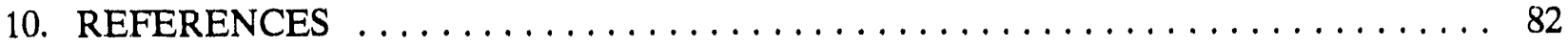




\section{LIST OF FIGURES}

Figure

Page

1.1 Conceptual representation of biological responses to toxicant exposure $\ldots \ldots \ldots \ldots \ldots 3$

1.2 Relationship between levels of biological organization and the relevance and time

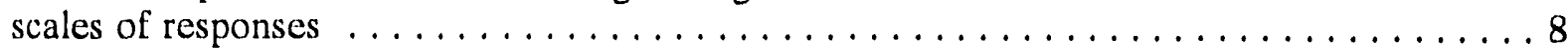

1.3 Linkages that provide support for attribution of a cause to an effect $\ldots \ldots \ldots \ldots \ldots$

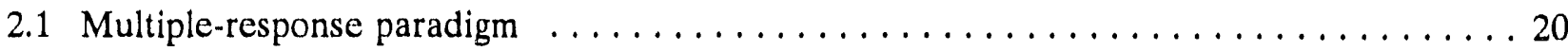

3.1 Segregation of integrated biomarker responses of female bluegills (Lepomis macrochirus) collected in the winter from four sites in a stream receiving industrial

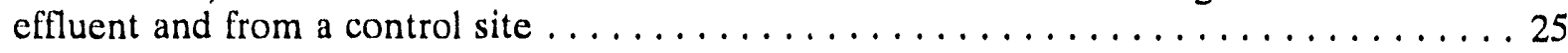

3.2 (a) Hypothetical map of contaminant pattern from sites containing different types of toxic chemicals. (b) Hypothetical map of biomarker responses detected in organisms inhabiting sites containing different types of toxic chemicals $\ldots \ldots \ldots 27$

5.1 Three-ticred hierarchy of environmental monitoring objectives for which biomarker responses can be a meaningful tool $\ldots \ldots \ldots \ldots \ldots \ldots \ldots \ldots \ldots$

5.2 Principal elements of a biomarker-based biomonitoring research program $\ldots \ldots \ldots 37$

5.3 Characteristics that can be associated with the Hazard Identification tier of the

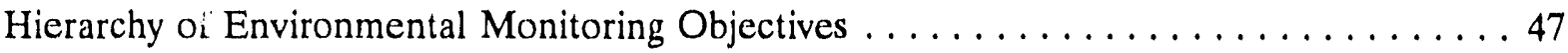

5.4 Characteristics that can be associated with the Hazard Assessment tier of the

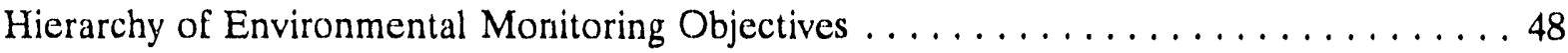

5.5 Characteristics that can be associated with the Risk Prediction tier of the Hierarchy of Environmental Monitoring Objectives . . . . . . . . . . . . . 49

5.6 Elements of a biomarker-based biomonitoring program with associated

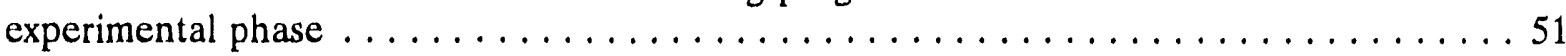

8.1 Proposed phases to be used in the gradual evolution and implementation

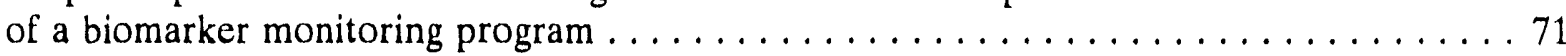




\section{LIST OF TABLES}

Table

$\underline{\text { Page }}$

4.1 Regulations and their relevance to biomarker study objectives $\ldots \ldots \ldots \ldots \ldots \ldots$

7.1 Biomarkers of genotoxicity/carcinogenicity $\ldots \ldots \ldots \ldots \ldots \ldots \ldots \ldots \ldots \ldots \ldots \ldots \ldots$ 


\section{PREFACE}

This document describes a strategy for defining specific objectives for biomarker studies and for designing and implementing a biomonitoring study that focuses on these objectives. In researching this subject, it became clear to the authors that the subject of biomarkers created a great deal of interest among scientists and regulators but that general acceptance of biomarkers as a tool for environmental protection was hampered by lack of a clear notion of how to develop and apply this approach. We intend this document to be a "user's guide" that lays out a logical scheme for applying biomarkers in environmental monitoring. In addition, laboratory and field research components needed to develop and validate fundamental understanding and interpretation of biomarker responses are also described, as is a strategy for evolution of a biomarker-based biomonitoring capability. The document is divided into sections intended to lead the reader to an understanding of how biomarkers can be developed and applied.

Section 1, an introduction, provides a general background on biomarkers, including their advantages and limitations. Many of the considerations and caveats are addressed in detail as elements to be considered in designing biomarker studies.

Section 2 is a discussion of conceptual paradigms for interpreting biomarker responses within a toxicological framework. The concept of dose-response relationships is toxicological dogma but may not be entirely appropriate for interpretation of biomarker responses in organisms exposed to multiple contaminants and environmental stresses. We propose an alternative paradigm for understanding the role of biomarkers in evaluating the biological hazards of toxic chemicals in the environment.

Section 3 is a discussion of statistical approaches that permit the use of biomarker responses to establish differences in the environmental health of organisms from sites of suspected contamination compared with that of animals from pristine reference sites. These methods make it possible to apply biomarker studies to problems of immediate concern while gathering data that will contribute to increased understanding and improved interpretation in the long run. 
In Sect. 4, many of the potential applications of biomarker-based monitoring are considered, especially in the context of federal legislation and regulatory needs. These many applications share a relatively limited number of specific objectives (e.g., identifying potential hazards or defining the geographic extent of a contamination hazard). This section is inte..ded to lead the potential user to a clearly defined statement of the objectives that can be achieved through biomarker-based biomonitoring.

Section 5 contains a conceptual strategy for designing a biomarker-based biomonitoring study. The general philosophical approach behind this section is that, although biomarkers can and are providing useful information in properly designed studies, development of consistent and intercomparable data bases from numerous studies will lead to improved understanding and more meaningful interpretation of biomarker responses. In this document we attempt to provide a unifying set of criteria and considerations for design of biomarker-based monitoring studies that will promote development of that understanding. Elements of a monitoring study are described, and a checklist of specific questions and considerations that must be included in the experimental design is provided. Although many elements and considerations are constant over a wide range of study objectives, certain elements must be tailored to achieve specific goals. Finally, we recognize that progress in understanding and interpreting biomarker responses requires a program of laboratory and field research; this research would be most productive and efficient if it were conducted in parallel with biomarker-based assessments of "real world" contamination problems. The elements and considera ${ }^{2}$ ' $n$ s to be included in research studies is outlined, and the relationship between application of biomarkers and research on biomarkers is indicated.

Section 6 touches on factors to be considered in the selection and prioritization of biomarkers.

Section 7 is a brief exploration of an application of biomarkers as additional endpoints in toxicity tests. Although this application is quite different from the use of biomarkers in environmental sentinel species, it may prove to be a very useful and informative adjunct to current bioassays. Biomarker responses may make it possible to use short-term bioassay systems to detect potential hazards that require a long time to be expressed (such as cancer). 
In Sect. 8 the implementation of a long-term research program designed to develop an validate a biomarker-based biomonitoring capability is considered. This implementation strategy proposes a gradual growth and evolution of field and laboratory studies that begins with simple systems and matures into a capability to interpret results of monitoring studies in more complex systems.

In summary, this document is intended to introduce the reader to the concepts of biomarker-based biomonitoring and to provide a logical pathway for designing, implementing, and validating a capability for using biomarkers for environmental protection. We hope that this will be accepted as a strategic guideline for international efforts in developing and implementing biomarker-based biomonitoring. This common strategy will help to focus research efforts and permit all researchers to benefit more directly from parallel research efforts in the United States and abroad. To promote this international consensus, this document also is intended as a "strawman" document for a proposed NATO Advance Research Workshop currently under consideration. If approved, this workshop will gather international experts to expand and hone the strawman to develop an internationally accredited "how-to" manual for biomarker studies and thus develop within the international scientific community a unified strategy for biomarker research. We hope that development of a common strategy also will hasten international acceptance of biomarker measurements as a tool for environmental assessment and compliance. 


\section{INTRODUCTION}

Biological monitoring is an informative, cost-effective, and logical complement to chemical monitoring of toxicants in the environment. The rationale for chemical monitoring is, after all, our concern for the potential threat thiat chemicals can pose to biological resources. Direct probing of biological responses to environmental contaminants is, therefore, a reasonable and necessary component of any environmental monitoring program. The concept of "biomarkers" has recently received considerable attention among environmental toxicologists as a new and potentially very powerful and informative tool for detecting and documenting exposure to and effects of environmental contamination.

It is the objective of this document to provide a conceptual framework for incorporating biomarkers as a component of a biological monitoring program for evaluating ecological exposures to contaminants. We provide a logical flow chart to assist in designing and implementing a biomarkerbased biomonitoring program. However, it is critical to recognize that (1) our current understanding of biomarker responses in environmental species is limited and (2) to achieve its full potential as a tool for environmental protection, a great deal of research will be needed to develop, validate, and interpret biomarker-based monitoring. This document describes an integrated laboratory-field approach for using controlled laboratory studies to supplement field monitoring programs to acquire fundamental information on biomarker responses in environmental species and on the role of environmental stresses on the expression of biomarker responses.

However, it is not necessary to achieve a complete understanding of all aspects of biomarker responses before biomarker data can be useful. What is critical is that researchers applying biomarker-based monitoring develop consistent and intercomparable data bases so that experience and understanding gained from one study can illuminate and improve interpretation of future studies. Progress in this research area requires (1) a coherent and logical strategy for acquiring experience and (2) a consistent and intercomparable data base. Such a strategy for progressing from monitoring of simple exposures through development of capabilities to predict long-term effects of contaminants is presented near the end of this document. It is our intention to provide a "user's guide" to designing biomarker-based monitoring programs now and to show how data from current studies can be supplemented by research to eventually develop the full potential of biomarkers as indicators of exposure and predictors of the consequences of environmental contamination. 


\subsection{BIOLOGICAL MARKERS OF ENVIRONMENTAL CONTAMINATION}

In general, biomarkers can be defined as measurements that indicate in biochemical or cellular terms that (1) an organism has been exposed to a chemical and (2) the magnitude of the host response. Various definitions have previously been proposed:

"Biological markers are indicators signaling events in biological systeins or samples" (NRC 1987).

"A biomarker is a change in a biological system that can be related to an exposure to, or effect from, a specific xenobiotic or type of toxic material" (Henderson et al 1989).

"Biological markers are measurements of body fluids, cells, or tissues that indicate in biochemical or cellular terms the presence and magnitude of toxicants or of host response" (NRC 1987).

"Biological markers may be defined as 'cellular, biochemical, or molecular alterations which are measurable in biological media such as human tissues, cells, or fluids.' For this presentation, I would add, 'and are indicative of exposure to environmental chemicals"' (Hulka and Wilcosky 1988).

"Biomarkers, broadly defined, are indicators of variation in cellular or biochemical components or processes, structures, or functions that are measurable in biological systems or samples" (NRC 1989).

A more appropriate definition and the one we propose to use is a modification of the definition used by the National Academy of Sciences (NRC 1989): "A biomarker is a xenobiotically induced variation in cellular or biochemical components or processes, structures, or functions that is measurable in a biological system or sample."

The concept of biomarkers is illustrated in F1.1. The objective in an environmental monitoring program is to avoid the unacceptable and often irreversible effects such as mass mortality, 


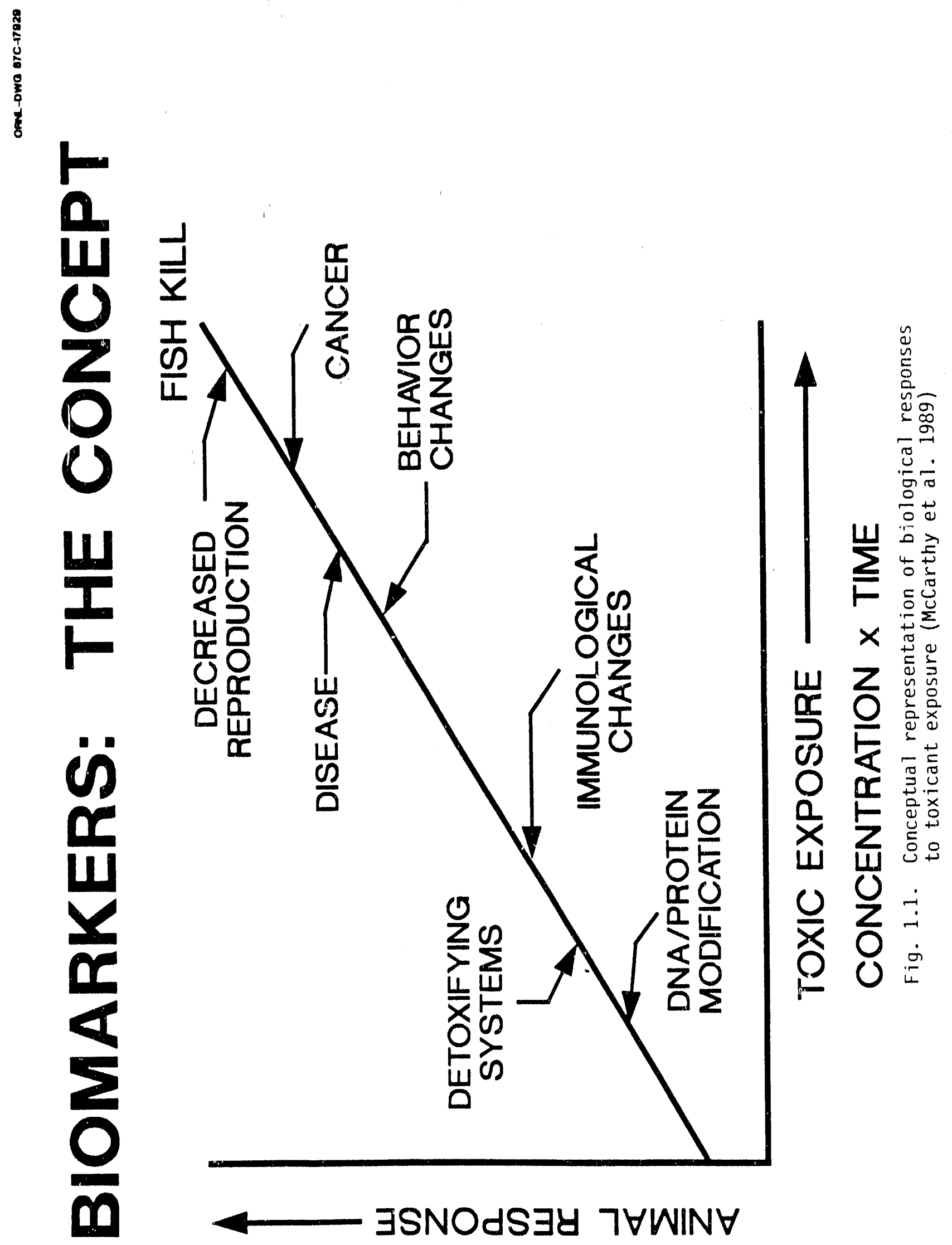


loss of commercially or ecologically impoitant species as a result of decreased reproduction, or increased susceptibility to disease. In measuring biomarkers, we regress down the conceptual sequence of animal responses to toxicant exposure to examine early responses that are causally related to, or predictive of, longer-term effects. Biomarkers are often divided into those that indicate exposure to chemical(s) and those that demonstrate toxic effects resulting from that exposire.

Biomarkers of exposure identify an exogenous substance within a system, the interactive product between a xenobiotic compound and endogenous components, or other event in the biological system related to exposure. These may include measures of internal dose or the biologically effective dose (i.e., the amount of material interacting with critical subcellular, cellular, and tissue targets or with an established surrogate) (NRC 1987).

Biomarkers of effect indicate an endogenous component of the biological system, a measure of the functional capacity of the system, or an altered state of the system that is recognized as impairment or disease. This includes an actual health impairment or recognized disease, an early precursor of a disease process that indicates a potential for impairment of health, or an event peripheral to any disease process but correlated with it, which is thus predictive of development of impaired health (NRC 1987).

Biomarkers of both exposure and effect can be useful in biological monitorin:. Because of the commonality of biochemical and cellular structure and function among organisms, biomarker approaches are potentially applicable over a broad range of species and across most ecosystem types. Additional information on biomarkers and their potential for application in evaluating euvironmental contamination is available in the proceedings of recent symposia and workshops (McCarthy and Shugart 1990; Huggett et al 1991).

\subsection{RATIONALE AND APPLICATION OF BIOMARKER INDICATORS}

Because the use of biomarkers is a relatively new approach to environmental monitoring, a general introduction to their advantages and limitations may be useful. In general, biomarkers are measured in organisms collected from (or confined to) sites of suspected contamination, and the results are compared with those from the same species collected from pristine reference sites. Measus ments 
of biomarkers provide a number of advantages that make it an informative and necessary adjunct to measurements of other indicators of contaminants in the environment, such as analyses of ambient chemicals, toxicity tests, and measurement of body burdens of toxic chemicals.

\subsubsection{Limitations of Existing Approaches to Environmental Assessment}

\subsubsection{Limitations of analyses of ambient chemicals}

Chemical measurements of environmental media are specific, quantitative, and exquisitely sensitive and precise. However, the biological significance of the chemical concentrations measured in air, water, soil, or food is not at all clear. We unferstand the toxic action of but a few of the thousands of chemicals in the environment and have almost no information on (1) the interactions of complex mixtures of chemicals or (2) the role of environmental stresses on an organism's susceptibility to toxic exposure. Furthermore, a chemical survey is a snapshot in time and space. Variations in concentrations over time resulting from intermittent releases of effluents by industries or from storm events, changes in winds, etc., cannot be accounted for without repeated analyses. Spatial patchiress of contaminant patterns also requires extensive and expensive sampling and chemical analyses.

Evidence of exposure in sentinel species provides a temporally integrated measure of bioavailable contaminant concentrations and is therefore much more relevant to the potential risk to the environment than is the analytically measurable concentration of contaminants in the soil, water, or air. Furthermore, mobile organisms integrate exposure over their spatial range and help to overcome problems of patchiness of ambient che micals. For analyses of exposure to complex mixtures of chemicals, biomarkers offer the potential for integrating the pharmacokinetic and toxicolcgical interactions within exposed organisms and expressing the cumulative impact of the exposure as a biomarker response measured at a site of toxic action.

\subsubsection{Limitations of toxicity tests}

Toxicity tests have proven very useful in detecting and quantifying adverse effects of individual chemicals, mixtures, effluents, and even sediments. However, many toxicity tests have limitations because they do not account for the effect of (1) chemical speciation in the environment, (2) kinetics 
and hysteresis in sorption of chemicals to sediment, (3) accumulation through foodchains, and (4) modes of toxic action that are not readily measured as short-term ( 7 to $21 \mathrm{~d}$ ) effects on survival, growth or reproduction. In situ monitoring of organisms collected or confined near discharges is a more realistic approach for determining the integrated exposure and effect of environmental pollutants. The combination of toxicity test data with demonstration of in situ effects in receiving bodies provides a compelling logical link between toxicity test endpoints and effects observed in the environment.

Biomarkers may also prove to be a useful addition to conventional toxicity tests to detect additional mechanisms of toxic action. For example, a fathead ininnow larval survival and growth assay could also be adapted to detect genotoxic effects if the larvae were tested for DNA alterations at the end of the regular test period.

\subsubsection{Limitations of measurements of tissue concentrations}

Measurement of tissue concentrations is highly recommended as an indicator of exposu $e$ to persistent compounds such as metals and certain classes of organic chemicals such as many polychlorinated compounds. However, when measuring tissue residues is not feasible, such as with compounds that do not readily bioaccurnulate (because of rapid metabolism, for example) or when measuring complex mixtures that require time- and cost-intensive analyses that may not identify all toxic chemicals, indirect measures of exposure (biomarkers) may be required or preferred. In addition, because the relationship between tissue concentrations and toxic effects is complex and not fully understood, biomarker measurements may indicate a response that is of toxicological significance.

\subsubsection{Biomarkers as Sensitive Indicators of Response to Environmental Jtress}

Animals in the natural environment are exposed to a variety of stresses including exposure to sublethal levels of contaminants, unfavorable or fluctuating temperatures, sediment loads, hypoxia, and food availability. Each of these factors, singly or together, can impose a considerable stress on physiological systems (Weidemeyer et al. 1984). Stress that exceeds the tolerance limits of organisms is obvious because it is usually lethal. Sublethal stress, however, is more insidious because adverse effects are generally manifested first at the suborganismal level. Depending on its severity, sublethal 
stress may load or limit physiological systems, reduce growth, impair reproduction, predispose to infectious diseases, and reduce the capacity to tolerate subsequent stress, including effects of toxic chemicals. At the population level, effects of stress may be manifested as reduced recruitment and compensatory reserves (Adams et al 1990).

Many of the biomarkers of effect, especially those at higher levels of biological organization such as histological, immunological, and physiologival/bioenergetic biomarkers, are sensitive and informative measures of the organism's response to cumulative stress from chemical, environmental, or pathogenic sources.

\subsubsection{Linking Toxic Exposure to Effects}

The ultimate indicators of ecological effects are adverse changes in exposed populations. However, population resjonses such as occurrence, abundance, and reproduction do not provide an indication of their cause. Correlation of population parameters with body burdens of chemicals and with sensitive and responsive molecular and biochemical biomarkers of exposure provides evidence of a causal linkage between exposure and effects. Correlations of population paraneters with ambient concentrations of chemicals or indices of chemical loading provide additional evidence of a causal link between the chemical releases into the environment and ecological effects. Nevertheless, population monitoring tends to be a rather insensitive indicator of effects because of the variability of anima! populations and the imprecision of field monitoring techniques.

Therefore, it is useful to monitor biomarkers of effects to provide a more sensitive and precise indicator of the nature and magnitude of effects and to gain insight into what caused the effect. The correlations between the biomarkers of effects and the indicators of exposure are expected to be better than those between the population monitoring parameters and the indicators of exposure.

Conceptually, measurement of biomarkers of exposure (including tissue concentrations of persistent chemicals) and biomarkers of effect develop evidence to statistically test hypotheses about the linkage between exposure to toxic chemicals and ecologically relevant effects. The rationale for this approach is indicated in Fig. 1.2, which illustrates the relationship between responses of various levels of biological organization and the relevance and time scales of the responses. Responses at the 


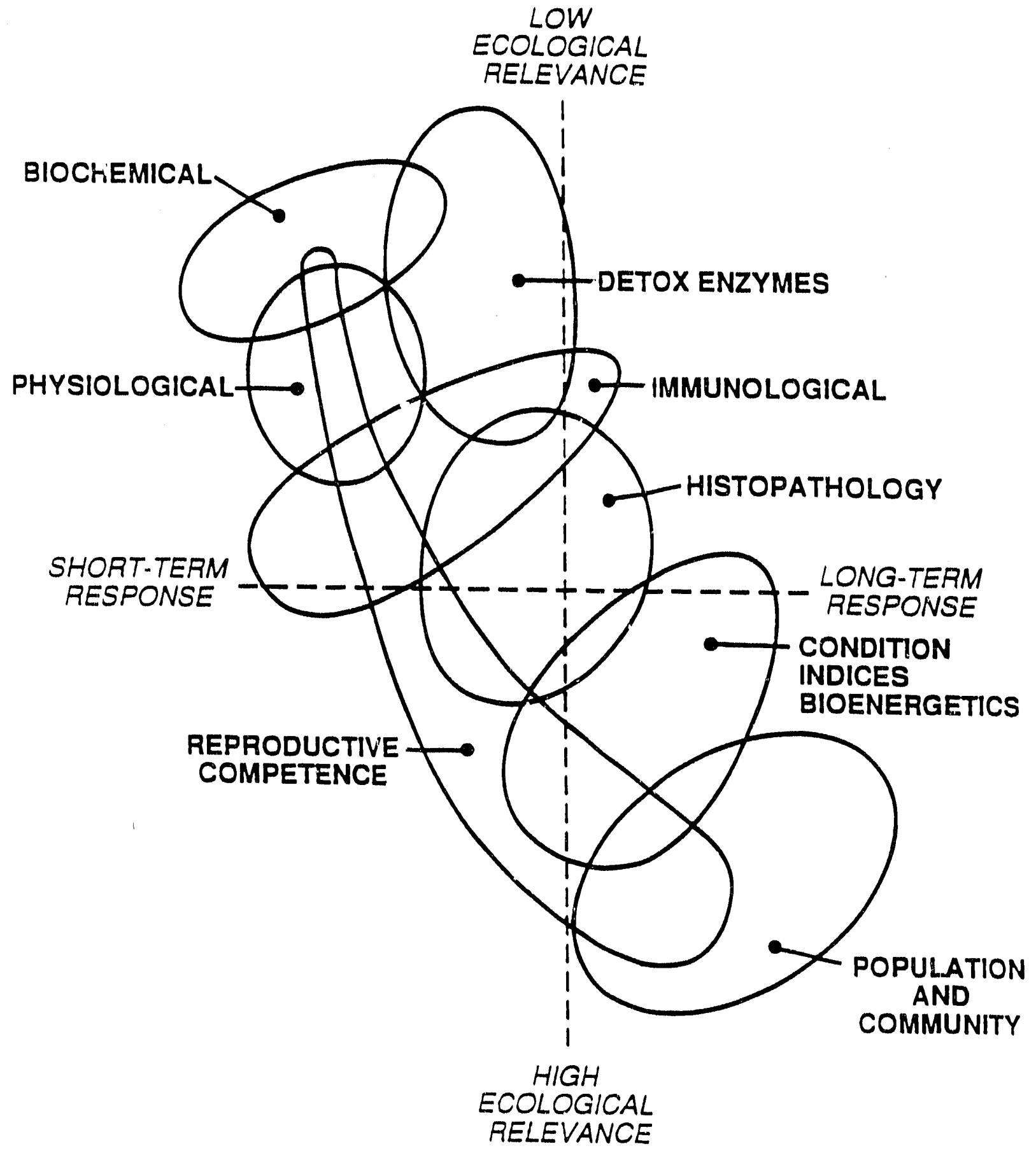

Fig. 1.2. Relationship between levels of biological organization and the relevance and time scales of responses (Adams et a 1. 2989). 
population and community level are highly relevant to ecological concerns but are slow to respond and difficult to attribute unequivocally to toxicants. In contrast, responses at lower levels of organization occur in shorter time frames and, consequently, can be more clearly linked to toxic exposure; however, it is difficult to relate these responses to effects at the community level. Our approach to biomarker-based biomonitoring is to measure responses at several levels of biological organization, including measurements both of (1) exposure to toxicants (generally responses in the upper-left quadrant of Fig. 1.2 but also including tissue burdens of chemicals) and (2) effects (generally the lower-right quadrant). Note that the division between responses that can be considered biomarkers of exposure vs biomarkers of effects is somewhat arbitrary. This blurring is a natural consequence of the interdependence inherent in the organization of biological systems. The goal in examining responses at these different levels of organization is to answer two critical questions:

1. Are organisms exposed to concentrations of toxicants that exceed the capacity of normal detoxication and repair systems?

2. If there is evidence of exposure, is the chemical stress affecting the integrity of the populations or communities?

Evidence of exposure from analyses of ambient chemicals, toxicity tests, and/or tissue concentrations and from biomarker responses of lower levels of biological organization provides an answer to the first question. In particular, the biomarkers of exposure indicate the biological significance of chemicals that may have entered the animal. (That is, did the chemical reach molecular and biochemical targets and cause detectible damage or induce a protective response?) The second question can be addressed by determining whether the responses to the toxicants are propagated up through successively higher levels of biological organization (biomarkers of effects and population parameters). If chemical exposure is responsible for a high-level ecological effect, responses should be apparent at intermediate levels of organization.

Alternatively, if data does not indicate any evidence of exposure or if biomarker responses indicate only minor effects in the most sensitive and responsive exposure parameters (e.g., genetic damage) but not at any higher levels of biological organization (e.g., histopathological evidence of neoplasia or tumors, or evidence of genetic abnormalities in gametes), community- and population-level effects could not be reasonably attributed to chemical agents. For this reason, a comprehensive approach 
also should examine alternative hypotheses for ecological effects to determine, for example, whether physical disturbance or other measures of habitat quality are better predictors of ecological response than are chemical-source or exposure parameters (Fig. 1.3). If the biomarker data indicate that some level of toxic effect is occurring but population parameters are better predicted by physical use or habitat quality, then multivariate statistics may be useful in determining whether effects of chemicals are contributing significantly to the observed population response (Suter 1990).

\subsection{General Indicators vs Chemical-Specific Indicators}

Some biomarkers are general indicators of exposure or effects, whereas others are specific responses diagnostic of particular chemicals or classes of chemicals. For example, inhibition of the enzyme aminolevulinic dehydrase is a specific indicator of exposure to lead, induction of the cytochrome P450 monooxygenase system is a specific response to organic contaminants such as polycyclic aromatic hydrocarbons (PAH's), and detection of DNA or protein adducts demonstrates exposure to specific chemicals. Conversely, other indicators provide evidence of responses to chemicals but do not identify the toxic agent responsible for the effect. For example, DNA integrity can be adversely affected as a result of chemical modification of DNA, physical damage from ionizing radiation or UV light, or inhibition of DNA repair systems. Similarly, induction of heat-shock stress proteins is a general indicator of response to a wide range of chemical or physical insults.

Both types of biomarkers are useful but may be most appropriately used as different tiers of a monitoring program. General indicators can be sensitive and cost-effective tools for broad spectrum screening studies to determine if there is any evidence of stress. If these general-indicator biomarkers respond in a manner that suggests a potential problem, a second tier of testing with more-specific biomarkers may be warranted.

\subsection{CONSIDERATIONS FOR DESIGNING AND INTERPRETING BIOMARKER RESPONSES}

Several factors must be considered in designing, executing, and interpreting a biomarker-based biomonitoring program. 


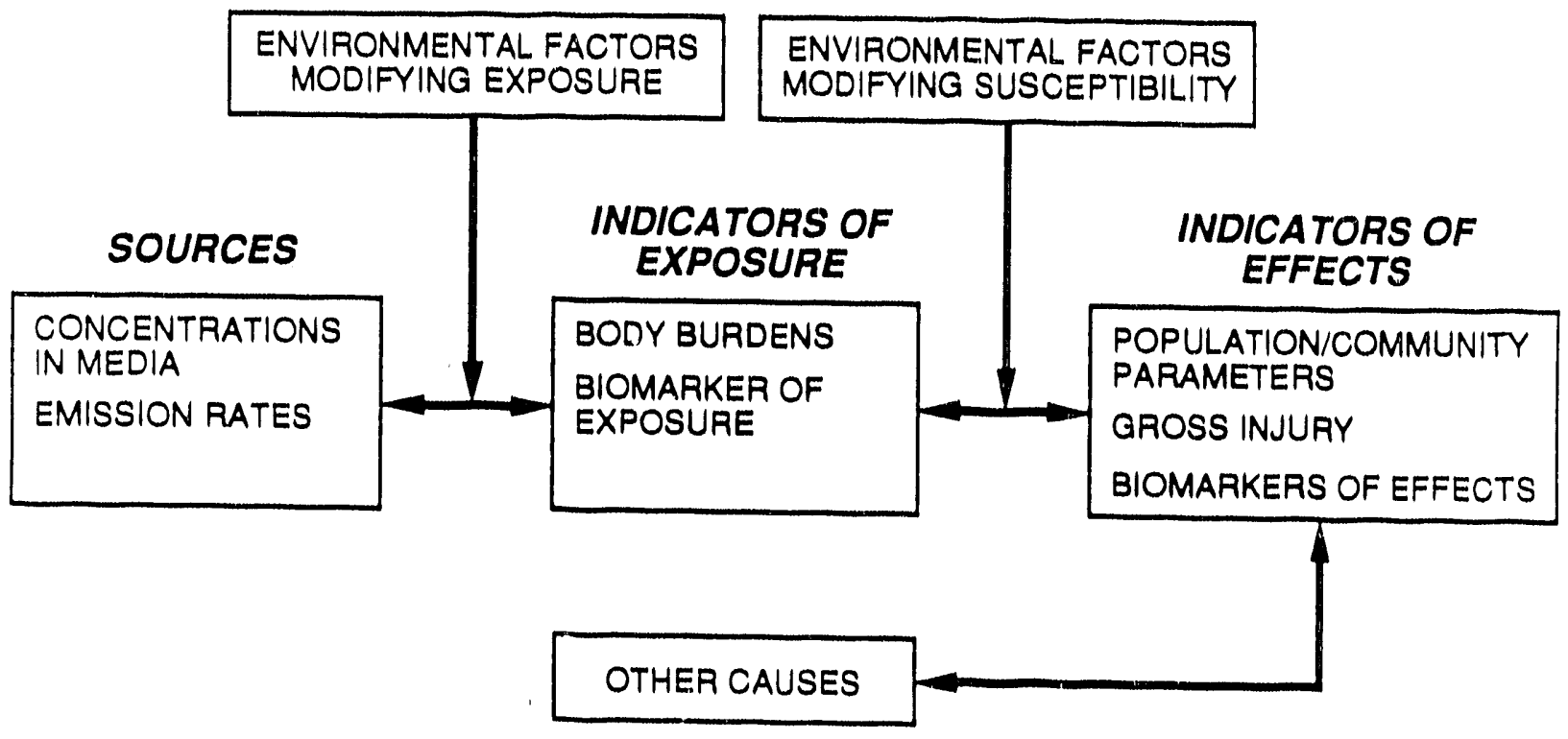

Fig. 1.3. Linkages that provide support for attribution of a cause to an effect (Suter 1990). 


\subsubsection{Selection of Species}

The routes of exposure and spatial range of organisms are among the criteria that should be considered in selecting the specific species to be collected for monitoring.

\subsubsection{Routes of Exposure}

The habitat and food preferences of sentinel species is an important factor that may aid in identifying the sources and routes of exposure. Fish and other aquatic species are exposed through water and sediment, and comparison of water-column vs sediment-associated species can distinguish the contribution of exposure from these media. Similarly, herbivorous rodents, such as volis and some mice, provide information on different routes of exposure than do muskrats and some shrews that dig in the soil (e.g., Loar et al. 1988; Talmage 1989).

In general, for chemicals such as metals that do not display biomagnification, physical positioning in the environment may be more important than trophic position in deterinining exposure. Typically, soil- or sediment-associated organism display the greatest tissue concentrations of contaminating metals and may be most useful for measuring biomarkers of exposure to metals (Martin and Coughtrey 1982). For compounds, such as persistent lipophilic chemicals such as polychlorinated biphenyls (PCBs) and PAHs, accumulation through trophic levels may be more important (Thoman 1981).

Confinements can limit access to defined routes, and confined animals can be used to test hypotheses about different routes of exposure. For example, animals can be provided with clean water and denied access to surface water, or vegetation can be removed from an enclosure and the animals provided with uncontaminated food. Farm animals or domesticated animals that drink well water can serve as sentinels of groundwater quality, and house-bound pets can be sentinels of exposure to indoor air pollutants; obviously, nondestructive analyses using blood or urine would be required for evaluating the exposure of pets. 


\subsubsection{Spatial Range}

The area of the home range of an animal sentinel needs to be matched to the size of the study site and the degree of geographic resolution required for a particular study. For example, voles, which range within an area $-20 \mathrm{~m}$ on a side, were useful for studying a small site such as Love Canal (Christian 1983). Larger sites might permit the use of rabbits or groundhogs, which range over acres and therefore integrate exposure over a wider geographic area. Bluegill sunfish have been useful in our studies in contaminated streams because tag-recapture studies have demonstrated that they confine themselves to a $100-\mathrm{m}$ reach in streams (Loar et al. 1988). Sessile animals, such as clams or mussels, provide excellent spatial resolution and may aid in the identification of "hot spots" of contamination. The selection of mobile or sessile species will depend on the specific objectives of the study. Animals confined at specific locations also can be used to increase the degree of geographic resolution in observations, to confirm and test hypotheses about the location of contaminants, and to test for the presence of localized "hot spots" of pollutants.

\subsubsection{Effects of Physiological and Environmental Variables}

Responses of many candidate biomarkers can vary depending on physiological and envirınmental factors such as age, sex, reproductive condition, temperature, and food availability (Jimenez et al. 1990). This caveat does not prevent the successful use of biomarkers, but it must be recognized that routine application of many biomarkers will require a fundamental understanding of the effect of these biological and environmental variables on the biomarker response to chemical exposure. Minimally, if responses of organisms from different contaminated and reference sites are to be compared, organisms should be collected so that seasonal and internal physiological influences are similar at the different sites. For example, responses of poikilothermic organisms collected in winter cannot be compared with those from even the same species collected in summer; likewise, for some indicators, responses of reproductively active females should not be compared with those from males or immature females (Jimenez et al. 1990; Loar et al. 1988). 


\subsection{GENERAL LIMITATIONS OF BIOMARKERS}

\subsubsection{Limited Validation}

It must be recognized that the developmert, application, validation, and interpretation of biomarkers is a relatively new field. With few exceptions, even the use of those blomarkers considered to be well understood and validated lacks a data base and historic track record comparable to more traditional methods to indicate exposure, such as analysis of chemical concentrations or standard toxicity tests.

Given the current status of our experience in applying and interpreting blomarker-based biomonitoring, many if not most biomarkers must currently be considered as qualitative indicators of exposure and effects and the significance of biomarker responses must be interpreted within the context of how they correlate with the better-documented exposure indicators. Nevertheless, biomarkers can provide valuable and informative data that will corroborate and extend other indicators such as chemical concentrations in the environment. More important, the significant longtarm advantages that biomarkers offer for monitoring environmental contamination counterbalances the limitations their currently modest data base imposes in the short term.

\subsubsection{Lack of Uniform Experience Across Ecosystem Types}

The majority of research on field evaluation of biomarkers in animals has focused on marine and aquatic systems. In the terrestrial environment, biomarker measurements in animals has been mostly limited to birds, although extensive biomedical laboratory research with rodents and rabbits suggests that biomarker approaches would be successful with other terrestrial animals. Nevertheless, data bases on field evaluation of biomarkers in terrestrial animals is limited.

In plants, more information is available for terrestrial plants than for aquatic macrophytes or algae. However, within terrestrial plants, most research has centered on effects of gaseous pollutants $\left(\mathrm{NO}_{x}\right.$ $\mathrm{SO}_{2}$, and ozone), and there are only a few biomarkers in plants that respond to toxic environmental pollutants. 


\subsubsection{QAVC and Cost Considerations}

Many biomarkers are still the object of research rather than tools of research. Methods have not been standardized, and many biomarker assays require fairly sophisticated research equipment. The problems of standardization of techniques and development of Quality Assurance/Quality Control procedures is currently being addressed by the American Society for Testing and Materials (ASTM), but it is likely to take a few years before definitive standards are developed and accepted by ASTM. Many biomarker meihods could be easily simplified and costs decreased by economy of scale and by introduction of automated clinical equipment. For example, research-grade spectrophotometers and fluorometers are not required for enzyme biomarkers; these assays can be casily adapted to highly automated centrifugal analyzers that are routinely used for human and veterinary blood chemistry profiles. Likewise, development of monoclonal antibodies could replace sophisticated and timeconsuming quantification of meta'solites or proteins with simple, quick (even field-portable) ELISA (enzyme-linked immunosorbent assay) kits. Until very recently, there has been little or no impetus for implementing these improvements, but the surge of interest in applying biomarkers suggests that this situation will change in the near future.

\subsection{SUMMARY}

Evaluating the exposure of biota to anthropogenic chemicals in the environment and determining the effect of that exposure on the ecological iniegrity of the environment is complex because of (1) the diversity of possible routes of exposure; (2) the large differences in the bioavailability of contaminants associated with these different media; (3) the complexity of molecular, biochemical, and toxicological interactions within organisms exposed to complex mixtures of chemicals; and (4) the often long latent period between exposure and manifestation of a population- and community-level response.

Measurement of biomarker responses in organisms from sites of suspected pollution can address many of these problems by identifying and assessing contaminant-associated risks to ecological resources. This can be accomplished because

1. biomarker measurements integrate exposure from different routes of exposure over time and geographically over the spatial range of the sentinel species; 
2. biomarker responses integrate the pharmacokinetic and toxicological interactions within organisms exposed to mixtures of contaminants;

3. biomarkers are generally rapid responses to toxicant exposure and thus may provide an early warning of long-term effects; and

4. biomarkers can help attribute an ecological effect to contaminant exposure by providing evidence that organisms are (or are not) exposed to levels of contaminants that exceer the capacity of normal detoxication and repair systems.

Thus, one of the more important advantages recommending development and application is biomarkers is their utility as a tool to diagnose the impact of chemical stress on biota and thereby to help in protecting ecological resources. 


\section{CONCEPTUAL PARADIGMS FOR EVALUATING BIOMARKER RESPONSE}

In recent years, technological advances have made it possible to detect and quantify biological markers that were previously unknown or below the levels of detection. Although strategies for research and evaluation of these responses are being developed in various fields of study (e.g., environmental exposure/effects, chemical risk assessment, and human epidemiological studies), openminded discussions and debates concerning the proper criteria to use in evaluating and interpreting biomarker responses have not kept pace with these technological advances. Presented here is a discussion of (1) the dose-response concepts currently used as a guide in evaluating biomarker response and (2) a newly emerging concept based on multiple responses.

Several biomarkers are measurements of cellular or physiological processes that are normal components of an organism's attempt to maintain a constant internal balance or to deal with normal metabolic processes [e.g., mixed function oxidase system (MFO) and oxygen-scavenging radicals]. The measured values of these processes will fluctuate within some normal range within an organism. If the organism is exposed to a xenobiotic contaminant, it may respond to this exposure by compensatory increases or decreases in one or several of these cellular or physiological processes. A measure of this abnormal increase or decrease in the cellular or physiological process is the biomarker. The challenge is to continue to develop the technology to detect changes in these cellular and physiological processes to be able to determine when this measurement has exceeded the values of normal homeostasis and to apply this information in the assessment of environmental health and risk analysis.

The dose-response model that is the generally accepted paradigm for evaluating the usefulness of a biomarker has some very beneficial uses but may not be the only paradigm for this purpose. Doseresponse is a concept formulated for controlled laboratory studies in which an organism is exposed to a single chemical and a response (biomarker) is measured over a range of doses. Information obtained from these laboratory responses are then extrapolated to organisms living under natural environmental conditions. The accuracy of this extrapolation has been the subject of much concern. Natural populations are not usually exposed to a single chemical but rather to mixtures of chemicals anci are subjected to many stresses not normally encountered in laboratory studies (e.g., heat and cold stress, nutritional and energy deficiency, and predator/prey stress). These variables greatly confound 
the extrapolation of laboratory studies to field situations. An organism under natural field conditions may respond quite differently to exposure than an organism in a laboratory situation. In fact, individuals within the same population may respond differently to the same exposure scenario, depending on individual idiosyncracies, genetic variability, varying states of nutrition, and varying microenvironments.

It is clear that interacting stresses and multiple exposures alter biomarker responses. For example, fish exposed to 3-methylcholanthrene (3-MC), a potent inducer of the MFO system, and then to benzo[a]pyrene $(\mathrm{BaP})$, a model genotoxic agent develop twice the number of DNA adducts as do fish receiving the same dose of $\mathrm{BaP}$ without 3-MC (McCarthy et al. 1989). The 3-MC stimulates the MFO system, which is responsible for metabolizing the BaP to the reactive intermediate that covalently modifies the DNA. Environmental stresses also have been known to produce analogous effects. Fish exposed to an aqueous solution of $\mathrm{BaP}$ and stressed with a rapid increase in temperature exhibited fivefold higher levels of DNA strand breaks than did fish exposed to the same dose of BaP without any temperature stress (Shugart 1990). Natural physiological processes also affect biomarker dose-response relationships; elevated concentrations of estradiol assnciated with female reproductive activity reduce contaminant-induced increases in the MFO system (Jimenez et al, 1990). With - sny one of these exposure scenarios, dose-response relationships are expected, but each set of exposure conditions appears to produce a different relationship.

Questioning the general applicability of a dose-response paradigm is not toxicological heresy; it is simply recognition that the naturai environment violates the underlying premise of good experimental design (i.e., that the effect of a single experimental variable-dose of toxicant-be evaluated in isolation). If all other variables (including the extent and type of environmental stress) were held constant, a reasonable dose-response relationship would be expected even in a natural environmental exposure. An organism stressed by multiple contaminants or ecological events would be expected to express progressively greater biomarker responses to increased doses of a single toxicant; however, that dose-response relationship may be quite different than the dose-response relationship exhibited by an individual of the same species that is free of additional stresses (or afflicted with different stresses). In the natural environment, it is unlikely that a single unique dose-response relationship exists that can relate a biomarker response to a dose of a chemical. In nature, it is more reasonable 
to think in terms of a family of dose-response relationships, each corresponding to different combinations and permutations of interacting chemical and environmental stresses.

These considerations make it difficult, and possibly inappropriate, to interpret biomarker responses either as a quantitative assessment of the exposure (dose received) or to predict the ultimate consequence of the exposure (e.g., deati, reduced reproduction, or neoplasia). Therefore, attempts to use laboratory-derived relationships to infer, for example, a dose of BaP to which a fish is exposed in nature based on the level of adducts measured in the wild animal's DNA may be intellectually attractive but are unlikely to be realistic or useful in most environmental situations.

Another concept or interpretation of biomarker responses is postulated and may serve as a more appropriate concept for designing biomarker research and interpreting the results of biomarker studies. The premise of this concept is an expansion of ideas recently published by Depledge (1989) to include aspects of biomarker studies. The basic scenario is that an organism in its natural environment maintains homeostasis (Fig. 2.1). As that organism is exposed to xenobiotic contaminants, physiological compensatory mechanıs ms become active, an increase/decrease in one or more physiological processes or functions occurs, and/or structural changes occur. All of these responses are biological markers (biomarkers) of response to the contaminant and, as such, indicate that exposure has occurred. If the exposure continues or increases, the organism's compensatory mechanisms become overwhelmed, damage occurs, and physiological repair mechanisms become active. At this point and with increased exposure or dose, additional biomarkers (e.g., reduced body weight, increased liver, spleen, and adrenal weights) are more prevalent and the susceptibility to disease and/or decimating factors (predation, freezing, starvation, and stress) increases. Under natural environmental conditions, as an organism progresses through these phases (homeostasis, compensatory response, and repair response), the energy required for normal maintenance is reduced as energy is needed for compensatory response and repair. The organism becomes progressively "weaker" and will most likely be quickly eliminated from the population. Therefore, in situ surveys of animal populations may not normally detect abundant organisms in a diseased condition even though exposure and effects have occurred. 


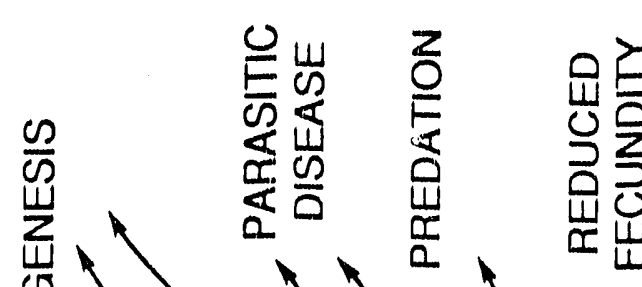

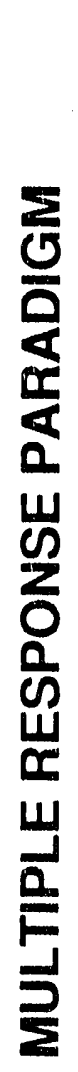
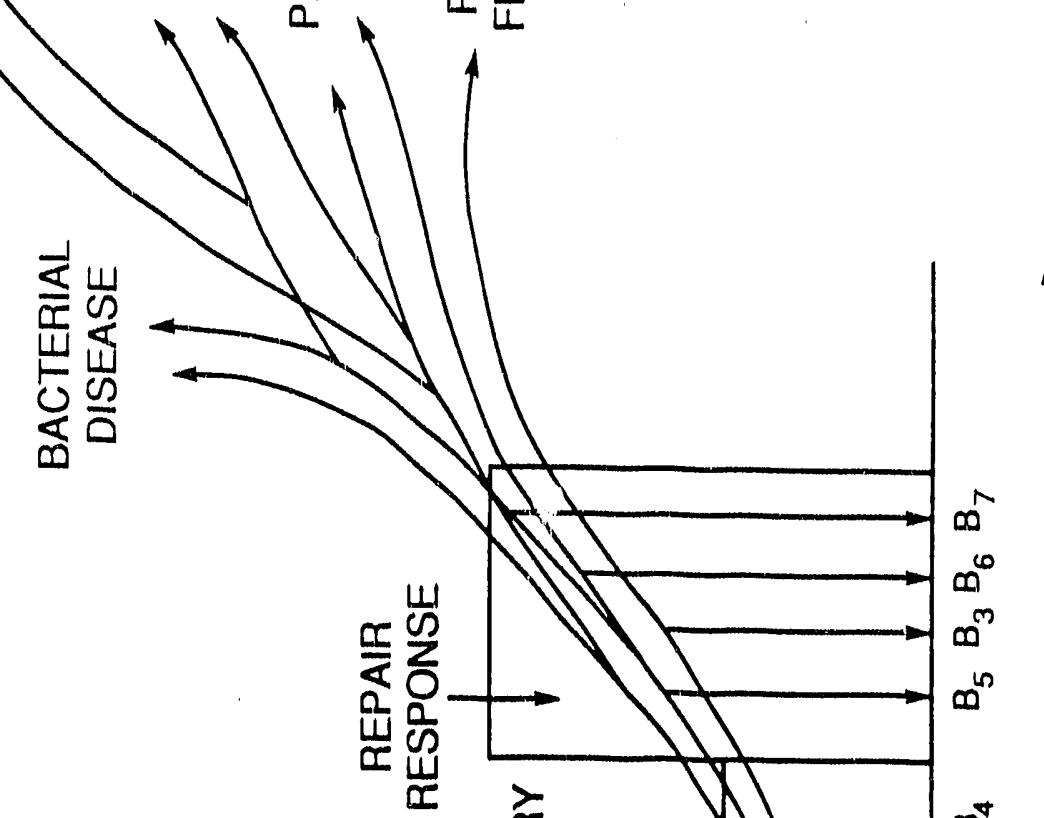

ธิ㇒

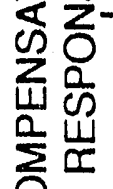

$\sum \frac{w}{x}$

ठํ.

-

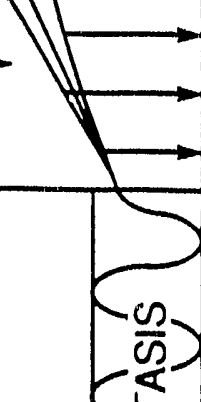

$\infty^{\sigma}$

$\infty^{\infty}$

$\infty$

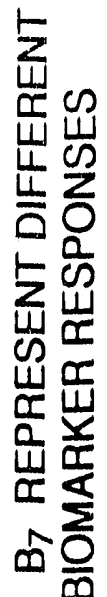

क

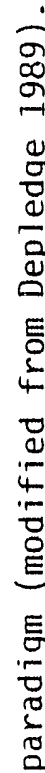

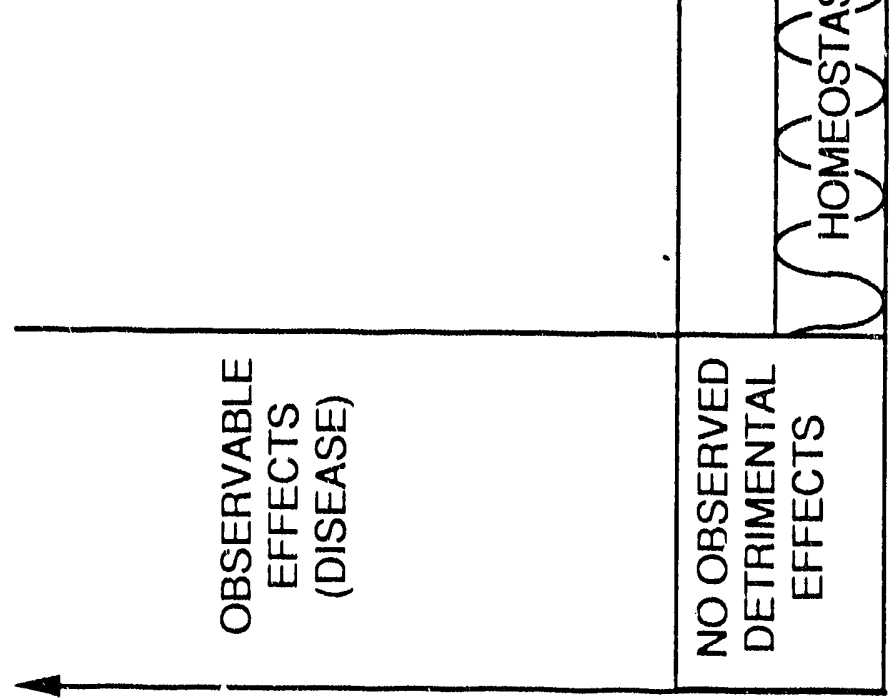

SUOLOVJ ONIL $\forall W I O \exists O O \perp$

A $\perp 7 \mid$ IIIdZOSNS ONIS $\forall \exists Y O N I$ 
In this multiple-response paradigm (illustrated in Fig. 2.1), the goal of blomarker measurements is not to quantitatively define the dose of different toxicants to which an organism is exposed but rather to determine where a population of organisms is located in the continuum between normal homeostasis and disease. Responses of a suite or battery of biomarkers serve as indicators of whether the animal is challenged but readily coping with toxicant stress (compensatory phase) or is being damaged and is having to expend resources to repair that damage. The goal of biomarker research is to identify which biomarker responses correspond with different levels of departure from normal homeostasis.

This paradigm has a number of useful features for interpreting biomarker responses to evaluate environmental health. It recognizes and accounts for the multiple chemical and ecological stresses to which organisms are exposed and expresses the net cumulative exposure in terms of a functional assessment of the overall health of an organism (and the population as a whole). Nevertheless, information may still be available that can help to determine the chemical agent responsible for any major toxic action; for example, induction of the MFO system is still indicative of exposure to organic contaminants. In essence, we are directly probing the biological system to determine the extent of a "health impact" of the contaminant exposure and expressing that information in terms that are relevant to environmental protection. The motivation for either chemical or biological monitoring is concern about organism and population health, not abstract quantification of the number amount or type of molecules entering an organism. Using biomarkers, we probe the toxic response directly within the organism, thus yielding much more powerful and meaningful information than does chemical monitoring. Furthermore, this approach makes it unnecessary to tie a specific consequence to a given exposure of toxicant(s), such as how many hepatic carcinomas will result if organismis hrve a certain level of DNA adducts or how many animals will succumb to a disease if their MFO levels are elevated by some percentage above background? It is unlikely that these questions can ever be reliably answered, even in a laboratory setting.

In many ways, the process of development and expression of a disease state shares many of the attributes of a chaotic system. In chaotic systems, small changes in initial conditions can greatly alter the final outcome. Thus, the ultimate "disease" the organism might develop can be affected by specific conditions at the time of exposure and interactions occurring over a period of time after exposure. Weather patterns are described as chaotic in that we cannot predict next year's weather 
from today's data. Likewise, we may not be able to predict that a certain level of adducts, in conjunction with some level of MFO induction, will result in a specific tumor incidence in a population. An abundant food supply may permit the organism to repair damage, or predation may eliminate the weakened animal before the tumor develops. However, the pattern and magnitude of biomarker responses may enable us to determine that the organisms are far from homeostasis and are closer to responses observed in populations of animals that are clearly in poor health (or that subsequently develop poor health with continuing exposures).

Both the dose-response and the multiple-response paradigms can provide useful information for evaluating exposure and effects of contaminants, and research needs to provide data that can be interpreted within the context of both models. Alternative hypotheses can be evaluated in laboratory and field research to determine which paradigm is most appropriate and useful. The dose-response paradigm would predict that biomarker responses will be well correlated with quantitative exposure (dose) and that dose will be predictive of some ultimate disease state. In contrast, the multipleresponse paradigm would predict that biomarker responses, while correlated to at least some extent with dose, will also be correlated with other stressors (multiple contaminants or environmental stress). Furthermore, the biomarker responses will have a much higher correlation with expression of some disease state or functional impairment than does the dose of a toxicant to which the organism is exposed.

Currently, data is not available to judge which paradigm is preferable, and information to make quantitative assessments of environmental impact based on biomarker responses is lacking. Our current state of knowledge does permit us to use biomarkers as qualitative or semiquantitative indicators of degree of exposure to deleterious agents. Our challenge is to build from this existing and valid application of biomarkers to develop a data base that enable us to evaluate these (and perhaps other) paradigms and to parameterize the more effective model to provide a more quantitative and predictive assessment of environmental contamination. 


\section{STATISTICAL EVALUATION AND INTERPRETATION OF BIOMARKER RESPONSES}

\subsection{DIFFICULTIES IN EVALUATING BIOMARKER RESPONSES}

Section 2 outlined the conceptual paradigms that provide an intellectual framework for organizing and focusing long-term research efforts. These paradigms serve as goals for the ultimate realization of the full value and power of biomarkers. However, as noted, much more research and data collection is needed before environmental health can be evaluated based on biomarker responses interpreted in the context of these paradigms. However, biomarker-based biomonitoring currently can provide valid and useful information for environmental assessments. The evaluations are based on statistical comparisons of the relative magnitude and/or pattern of biomarker responses of organisms from sites of suspected contamination compared with those of similar organisms from pristine reference sites. Several statistical approaches will be discussed; however, it is important to recognize that statistical evaluations must be accompanied by insight into the pattern of responses among different types of biomarkers and the possibility of confounding interactions of environmental, physiological, or toxicological factors. These interactions may suggest changes in selection of data for analyses or may require changes in the design of the study. It should be emphasized that these difficulties do not invalidate the application of biomarkers. However, they do highlight the fact that application and interpretation of biomarker responses will require a basic understanding of how environmental and biological variables alter the responses of biomarkers to contaminant exposure. These variables need to be the subject of laboratory research, and consideration should be given to these factors in designing and interpreting statistical analyses of biomarker studies.

\subsection{ANALYSIS AND INTERPRETATION OF BIOMARKER RESPONSES}

A properly conducted biomarker-based biomonitoring study provides a large body of data, including information on chemical concentrations in tissues and media, as well as responses of a broad suite of biomarker responses at the molecular, biochemical, physiological and organismal level. How do we integrate and interpret the multiple variables measured as body burdens, biomarker responses, and adverse effects? The objectives of analysis include 
1. hazard identification-determining if there is evidence of bioavailable contamination at different sites,

2. hazard assessment-describing the quantitative and qualitative pattern in the responses and identifying groups of chemicals that may be involved, and

3. risik prediction-extrapolating the responses of the individual measurements to community-level eff ects.

Differences in the responses of animals collected from different sites can be compared statistically in several ways, the following among them.

Multivariate discriminant analysis-Hypothesis testing using multivariate discriminant analysis has been used to provide an integrated measure of responses of a large number of biomarker responses (Adams et all. 1988, 1990; Loar et al. 1988). This method can statistically evaluate similarities or differences between sites and identify those response variables that contribute the most to differences between sites. For example, this procedure could be used to demonstrate that the integrated responses of organisms from a suspect site were significantly different from those of organisms from reference sites suggesting contaminant exposure (see Fig. 3.1, for example). Furthermore, the analysis could also be used to establish that discrimination between the sites resulted primarily from toxic responses (DNA damage and induced MFO activity) rather than from bioenergetic factors (lipid content or condition indices), corroborating the role of toxicants in causing the differences between sites (Adams et al.. 1990).

State space analysis-Although it has been common practice to use hypothesis testing in the evaluation of environmental contaminant data collected in the field, this practice is questionable. Hypothesis testing is appropriate for testing effects when replication and random assignment of treatments is possible. However, in the evaluation of exposure to environmental contaminants based on responses of animals collected from different sampling sites, true replication and random assignment of treatments is not normally possible (Suter 1990). Therefore, evaluation and interpretation of results should probably be based on descriptive statistics rather than hypothesis testing. Staie space analysis establishes what constitutes a normal state for an organism or population, and determines the extent to which the suspect sites fall outside the "normal" state space. 

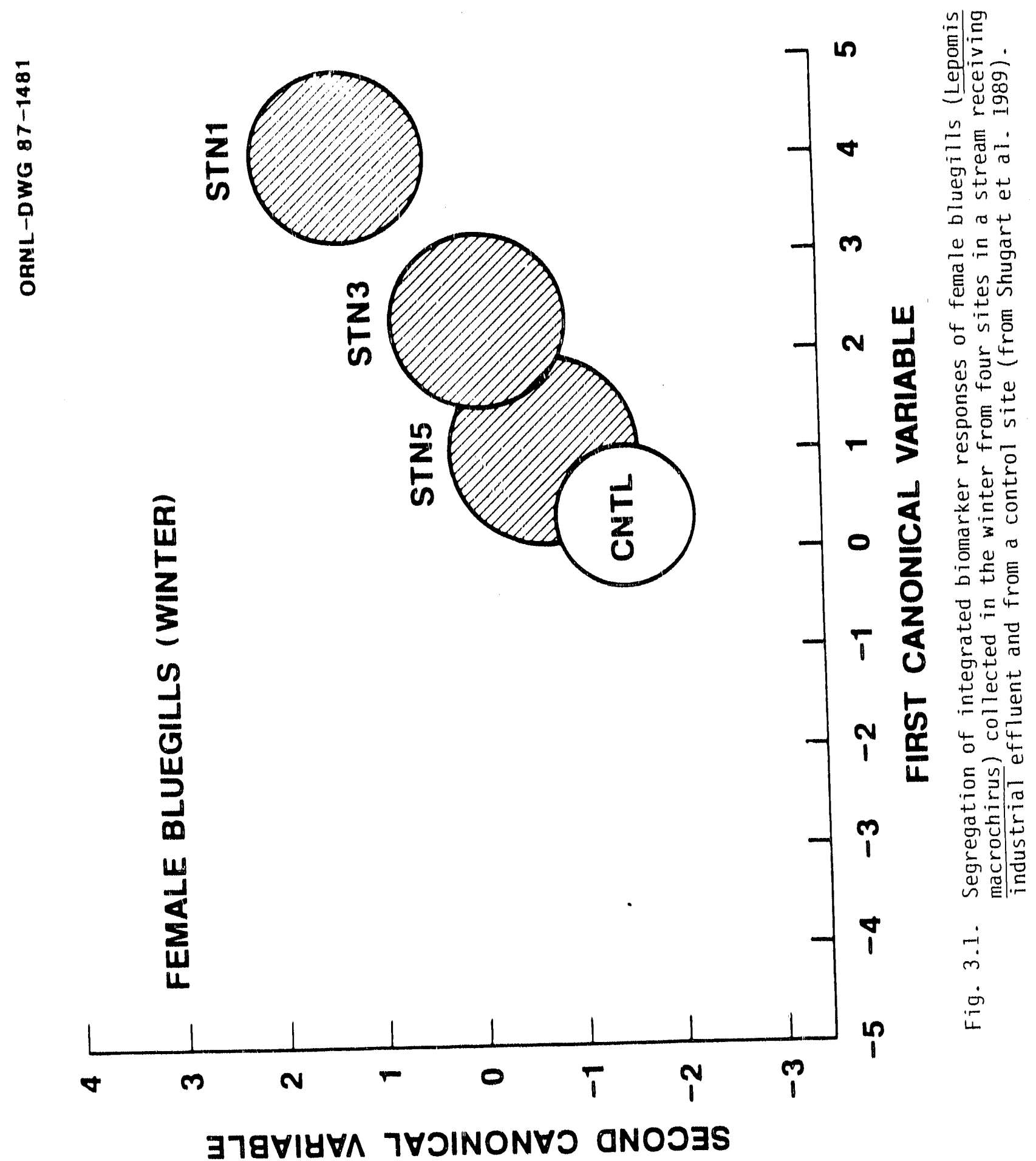
Multiple biomarker responses, body burdens, or community or population data at the reference sites establish the $n$-dimensional "normal" state space (where $n$ is the number of variables that were measured). Measurements at suspect sttes define the state space for those sites. The distance among sites can then be measured by Mahalanobis generalized distance (Johnson 1988;

Suter 1990). Discriminant analysis can also be used to determine which variable best accounts for differences among sites. The distinction between state-space analysis and multivariate analysis rests primarily on how the final outcome is interpreted. In hypothesis testing, differences between treatments are assigned objectively by statistical criteria. In descriptive statistics, expert judgement is required to rule whether a 10 or $50 \%$ difference in the state space between sites is an appropriate criteria. These evaluation criteria should be established early in the experimental process and will depend on expert opinions, previous field siudy results, laboratory studies, and other information available to the researchers.

Pattern or cluster analysis-The patterns of geographic distributions of body burdens and markers can be analyzed with multivariate classification procedures (cluster or pattern analyses) to group patterns of responses of the biomarkers and attempt to establish "fingerprints" characteristic of exposures to different types of contaminants. These analyses would also permit the responses of variables of body burden and biomarkers to be geographically mapped onto the hazardous waste site and adjacent population areas. The overall goal of this approach is (1) to determine whether differences in biomarkers and/or body burden in animals exposed to toxicants at a hazardous waste site and animals from a pristine site can be measured and (2) to discriminate quantitative differences in the concentrations and types of contaminants around the waste site. An expected result from such a hypothetical survey is illustrated in Fig. 3.2. Based on measurements of body burdens and biomarkers from various areas on this site, multivariate statistical analyses have grouped responses into hypothetical patterns or "fingerprints" and mapped them onto a geographic map of the site. The contaminant pattern at the Bear Creek Valley Hazardous Waste Disposal Site in Oak Ridge, Tennessee, serves to illustrate the point. This waste site has distinctly different patterns and types of contaminants in different locations on the site. The oil land farms have high levels of polynuclear aromatics (PNAs) from petroleum wastes. A solvent dump pipe area is heavily contaminated with solvents such as trichloroethylene. Further west, transformer fluids with PCBs were disposed of for several years. High levels of PCBs are expected in soils, in the oil retention pond, and in the 


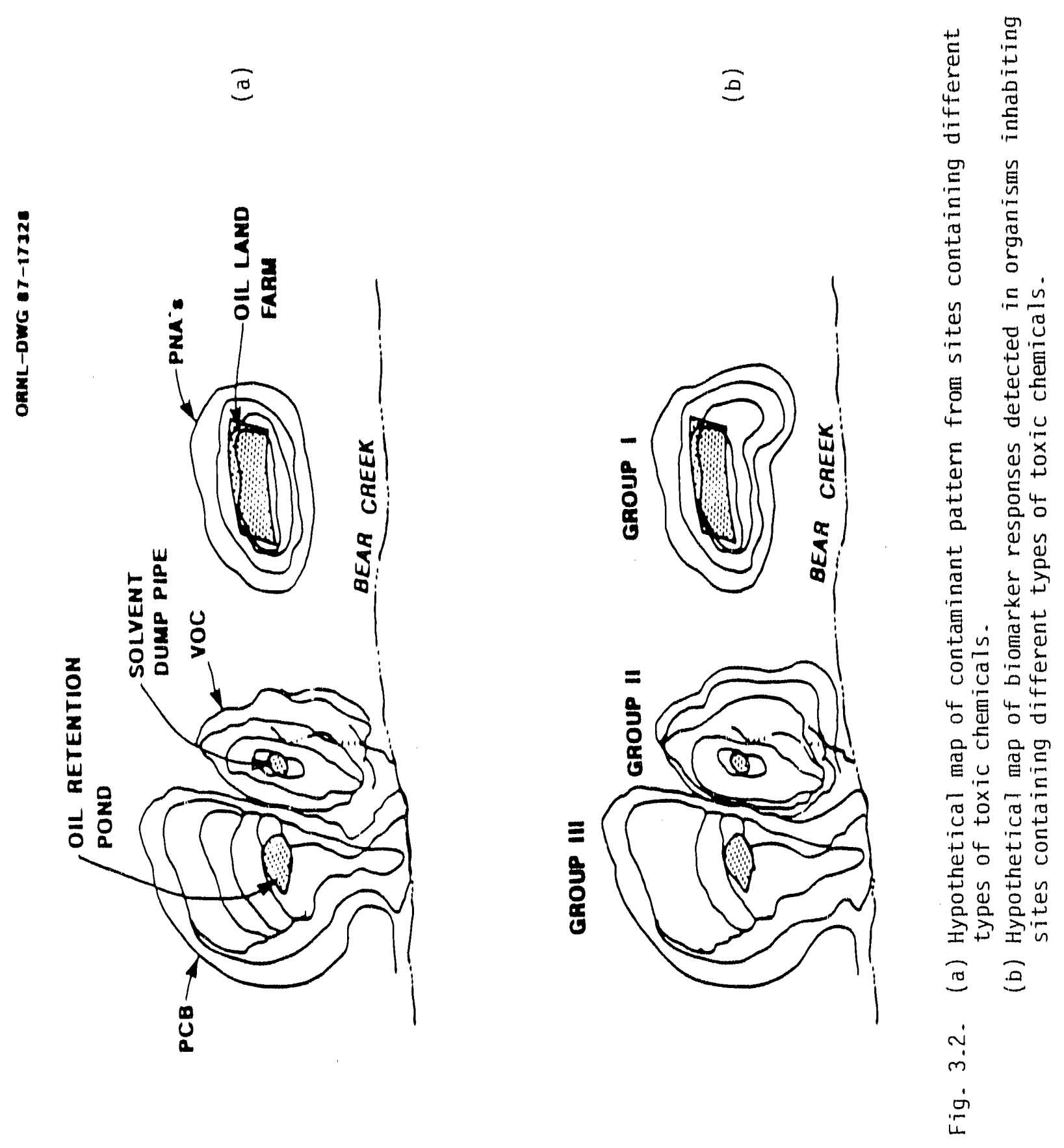


sediments of Bear Creek. Figure 3.2 illustrates the expected results of a hypothetical chemical survey of the area, expressed as isopleths of chemical concentrations. Figure 3.2 illustrates that we would ixpect different patterns or fingerprints of biomarker responses to be characteristic of the different classes of contaminants at the three locations, and it suggests a possible quantitative decrease in the intensity of the responses in animals further away from most highly contaminated locations. Superimposition of a map of chemical concentration and type on the map of biomarker responses would indicate sources of the mcst bioavailable contaminants and help guide remediation. 


\section{DEFINITTION OF APPLICATIONS AND OBJECTIVES OF B.X.)MARKER-BASED BIOMONITORING}

Biomarkers can be a cost-effective tool for a number of applications, most of which are motivated, directly or Indirectly, by regulatory concerns. These numerous regulatory applications devolve into a more limited and workable number of objectives that plovide the basis for focusing strategies for development and application of biomarkers. The objectives of these applications may include documenting exposure, determining the geographic extent of bloavailable contaminants, distinguishing relative degrees of contaminant-related stress at different sites, or predicting ecological risks to an environment. To design an appropriate and focused study, it is essential to consider different applications of biomonitoring and identify specific objectives for the study.

Table 1 illustrates how biomarker-based biomonitoring can be applied to a number of regulatory mandates. The objectives are organized within the context of a tiered system progressing from hazard identification (Is there any problem?), through hazard assessment (I know there is a potential problem, but just how bad is it?), and finally to risk assessment (What is the probability of an adverse ecological effect?). Within each tier, there are more-focused subobjectives; for example, we know there is pollution at a site and now wish to assess the geographic extent of that hazard.

This table is not intended to be all-inclusive; rather, it is intended to provide several familiar examples so that the reader can begin to extrapolate to other regulations and to identify objectives amenable to application of biomarker-based biomonitoring. The objectives indicated on Table 1 correspond to objectives used to define and design the specific aspects of a biomarker-based biomonitoring study (see Sect. 5). The following describes the relationship between applications and biomarker research objectives for several of the regulations listed in the table.

The Clean Water Act mandates that effluent discharges and receiving waters be tested for potential toxicity. Biomarkers can be used as endpoints in standard bioassays (see Sect. 6) to detect, for example, potential genotoxicity. Alternatively, biomarker responses in animals collected from waters near the discharge could be compared with those of animals from reference sites to establish potential toxic exposure. 
Table 1. Regulations and their relevance to biomarker study objectives.

\begin{tabular}{|c|c|c|c|c|c|c|c|c|c|c|}
\hline \multirow[b]{2}{*}{ Objective } & \multicolumn{10}{|c|}{ Regulation" } \\
\hline & $\begin{array}{l}\mathbf{C} \\
\mathbf{W} \\
\mathbf{A}\end{array}$ & $\begin{array}{l}\mathbf{F} \\
\mathbf{I} \\
\mathbf{F} \\
\mathbf{R} \\
\mathbf{A}\end{array}$ & $\begin{array}{l}\mathbf{R} \\
\mathbf{C} \\
\mathbf{R} \\
\mathbf{A}\end{array}$ & $\begin{array}{l}\mathbf{S} \\
\mathbf{A} \\
\mathbf{R} \\
\mathbf{A}\end{array}$ & $\begin{array}{l}\text { CR } \\
\mathbf{R} \\
\mathbf{C} \\
\text { LA }\end{array}$ & $\begin{array}{l}\mathrm{T} \\
\mathbf{S} \\
\mathbf{C A}\end{array}$ & $\begin{array}{l}\mathbf{O} \\
\mathbf{D} \\
\mathbf{A}\end{array}$ & $\begin{array}{l}\text { NP } \\
\mathbf{D} \\
\mathbf{E} \\
\mathbf{S}\end{array}$ & $\begin{array}{l}\mathbf{E} \\
\mathbf{M} \\
\mathbf{A P}\end{array}$ & $\begin{array}{l}\mathbf{N} \\
\mathbf{S} \\
\mathrm{T} \\
\mathbf{P}\end{array}$ \\
\hline \multicolumn{11}{|l|}{ Hazard Identlfication } \\
\hline Identify possible hazard at a point source & $\mathrm{x}$ & & $\mathrm{x}$ & $\mathbf{x}$ & & & $\mathrm{x}$ & $\mathrm{x}$ & & \\
\hline $\begin{array}{l}\text { Identify potentlal hazard for a nonpoint source of } \\
\text { pollution }\end{array}$ & & & & & & & & & $\mathrm{x}$ & $\mathrm{x}$ \\
\hline $\begin{array}{l}\text { Monitor temporal changes in contaminant exposure } \\
\text { (trends) }\end{array}$ & & & & $\mathrm{X}$ & & & & $\mathbf{X}$ & $\mathbf{X}$ & $\mathrm{x}$ \\
\hline \multicolumn{11}{|l|}{ Hazard Assessment } \\
\hline Describe geographlc extent of contamination & & & & $\mathrm{x}$ & & & $\mathrm{x}$ & $\mathrm{X}$ & $\mathbf{X}$ & $\mathrm{x}$ \\
\hline Assess the severity of exposure/effects & & $\mathbf{x}$ & & $\mathrm{x}$ & & $\mathrm{x}$ & $\mathbf{x}$ & $\mathrm{x}$ & $\mathrm{x}$ & $\mathrm{x}$ \\
\hline Identify critical routes of exposure & & $\mathrm{x}$ & & $\mathrm{x}$ & & & $\mathrm{x}$ & & $\mathrm{x}$ & $\mathrm{x}$ \\
\hline Identify chemicals contributing to exposure & $\mathrm{x}$ & $\mathrm{x}$ & & $\mathrm{x}$ & & & is & $\mathrm{x}$ & $\mathrm{x}$ & $\mathrm{x}$ \\
\hline Determine temporal trends in exposure and effects & & & & $\mathrm{x}$ & & & & $\mathrm{X}$ & $\mathrm{X}$ & $\mathrm{x}$ \\
\hline \multicolumn{11}{|l|}{ Risk Assessment } \\
\hline Attribute ecological effect to contaminant exposure & & $\mathrm{x}$ & & $\mathrm{x}$ & & & & $\mathrm{x}$ & $\mathrm{x}$ & $\mathrm{x}$ \\
\hline Predictions of long-term effects & & $x$ & & $\mathrm{x}$ & & $\mathrm{x}$ & $\mathrm{X}$ & & $\mathrm{X}$ & $\mathrm{x}$ \\
\hline
\end{tabular}

"CWA_Clean Water Act; FIFRA_Federal Insecticide, Fungicide, and Rodenticide Act; RCRA-Resource Conservation and Recovery Act; SARA-Superfund Amendment and Reauthorization Act; CERCLA-Comprehensive Environmental Restoration, Compensation, and Litigation Act; TSCA-Toxic Substances Control Act; NPDES-National Pollution Discharge Ellmination System; EMAP-Environmental Monitoring and Assessment Program; and NSTP-National Status and Trends Program. 
The Environmental Protection Agency's (EPA's) Environmental Monitoring and Assessment Program and the National Organic and Atmospheric Administration's (NOAA's) National Status and Trends Program seek to establish routine monitoring to assess the health of the environment and establish temporal trends in environmental quality. Biomarkers can be used in a screening mode (non-pointsource hazard identification) using very general indicators of toxic stress. Alternatively, biomarkers can help establish (1) that differences among sites are attributable to toxicant exposure, (2) the geographic extent of the contamination, and (3) temporal trends in either the extent or severity of contamination and may be useful in assessing the risk the contamination poses to the ecological resource.

The Federal Insecticide, Fungicide, and Rodenticide Act is the pivotal regulation for ensuring that the agricultural use of pesticides does not pose a threat to the environment. The enzyme biomarker cholinesterase can and has been used in screening areas where insecticides have been applied in agricultural uses. Monitoring of both wild and domestic species provides information on the severity and extent of exposure to these chemical compounds. This knowledge has lead to other uses of this biomarker. Under mandates from congress, the U.S. Army has been authorized to destroy stockpiles of chemical weapons (nerve agents). These agents can have similar but much stronger effects on cholinesterase activity than do the organophosphate insecticides (Carnes and Watson 1989). Therefore, cholinesterase activity has been proposed as a biomarker of nerve agent exposure during the destruction phase of these agents.

Biomarkers are sensitive indicators of exposure and effects and have multiple potential uses under the Superfund Amendment and Reauthorization Act and the National Pollution Discharge Elimination System. Both of these regulations rely on exposure and effect information to indicate problem areas and to measure the success of remedial action. Evaluation of biomarkers in organisms at sites that have already been identified as problem areas and suites of biomarkers at sites that may be suspect can provide valuable information needed by those making regulatory decisions under these acts. 


\section{CONCEPTUAL STRATEGY FOR DEVELOPMENT AND APPLICATION OF A BIOMARKER-BASED BIOMONITORING PROGRAM}

This section is intended as a "user's guide" to designing an environmental monitoring program using biomarkers. First, the objectives of the study are described in terms of a hierarchy made up of three tiers, which are defined by increasing levels of information about the existence and severity of the contamination problem at a site and by the need for additional information to evaluate the existance, extent, and severity of the problem. Although many components or elements of a properly designed monitoring study will be influenced by the specific objectives motivating the research, many elements and considerations in study design are generally applicable to a broad range of situations.

The logical pathway for designing an environmental monitoring study are indicated in Sect. 5.2. Each element in the pathway is dissected into a series of relevant issues that must be considered during the design and execution of the study. The relevant considerations are highlighted in the form of a "checklist" that calls attention to critical issues in design or implementation of a study. The checklist of critical issues continues in Sect. 5.3, but this section focuses on those elements of biomarker study design that are specific to particular applications or objectives.

Sections 5.2 and 5.3 approach the design of biomarker monitoring studies from an applied perspective and do not address the fundamental research that is needed to achieve a more complete understanding and interpretation of the biomarker responses. However, Sect. 5.4 builds off of the previous pathway for designing a monitoring study and describes a parallel conceptual pathway for devising and designing laboratory and field research to (1) improve basic understanding of biomarker responses; (2) elucidate the role of environmental and toxicological stresses on biomarker responses; and (3) confirm relationships between exposure, biomarker responses, and long-term adverse effects to individuals and populations.

\subsection{DEFINING STUDY OBJECTIVES}

Before any study is initiated, an objective or need for the study must be established. Generally, the objective is derived from a question (Has exposure occurred? What is the extent of exposure? What are the effects?), and the results of the proposed study should provide information needed to answer 
that question. Often, the objectives of the study are cierived from regulatory issues such as those discussed in Sect. 4.

Once a need has been established, an evaluation of information already available can be used to establish and define the objective of the proposed study. For example, will the study be intended for Hazard Identification, Hazard Assessment, or Risk Prediction? (Fig. 5.1).

\subsubsection{HAZARD IDENTIFICATION}

- First tier in the hierarchy of biomarker uses

- No readily identifiable chemicals of exposure

- Detection of biomarker responses indicating exposure

- Point, non-point, and/or trend analysis

Hazard identification is the first tier in the hierarchy of biomarker uses. Typical needs for which this tier would be used include the detection of biomarker responses that would indicate exposure to xenobiotics at point and or nonpcint sources of exposure. At this level in the hierarchy, it is assumed that little or no information is available on chemicals of exposure. An abandoned chemical or municipal dump or the discharge of effluent from a municipal wastewater treatment plant would create a situation in which questions of exposure might be raised. A short-term or trend study of exposure in the Chesapeake Bay or in some other valuable natural resource (e.g., Great Smoky Mountains National Park, Yosemite National Park) also may be included at this level of the hierarchy. Likewise, monitoring of status and trends of contamination of ecological resources [(such as EMAP or NSTP) see Sect. 4] fall within this category.

\subsubsection{HAZARD ASSESSMENT}

- Second tier in the hierarchy of biomarker uses

- Some chemical information is available

- A problem or suspected problem is known to exist and warrants additional study 


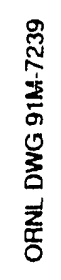

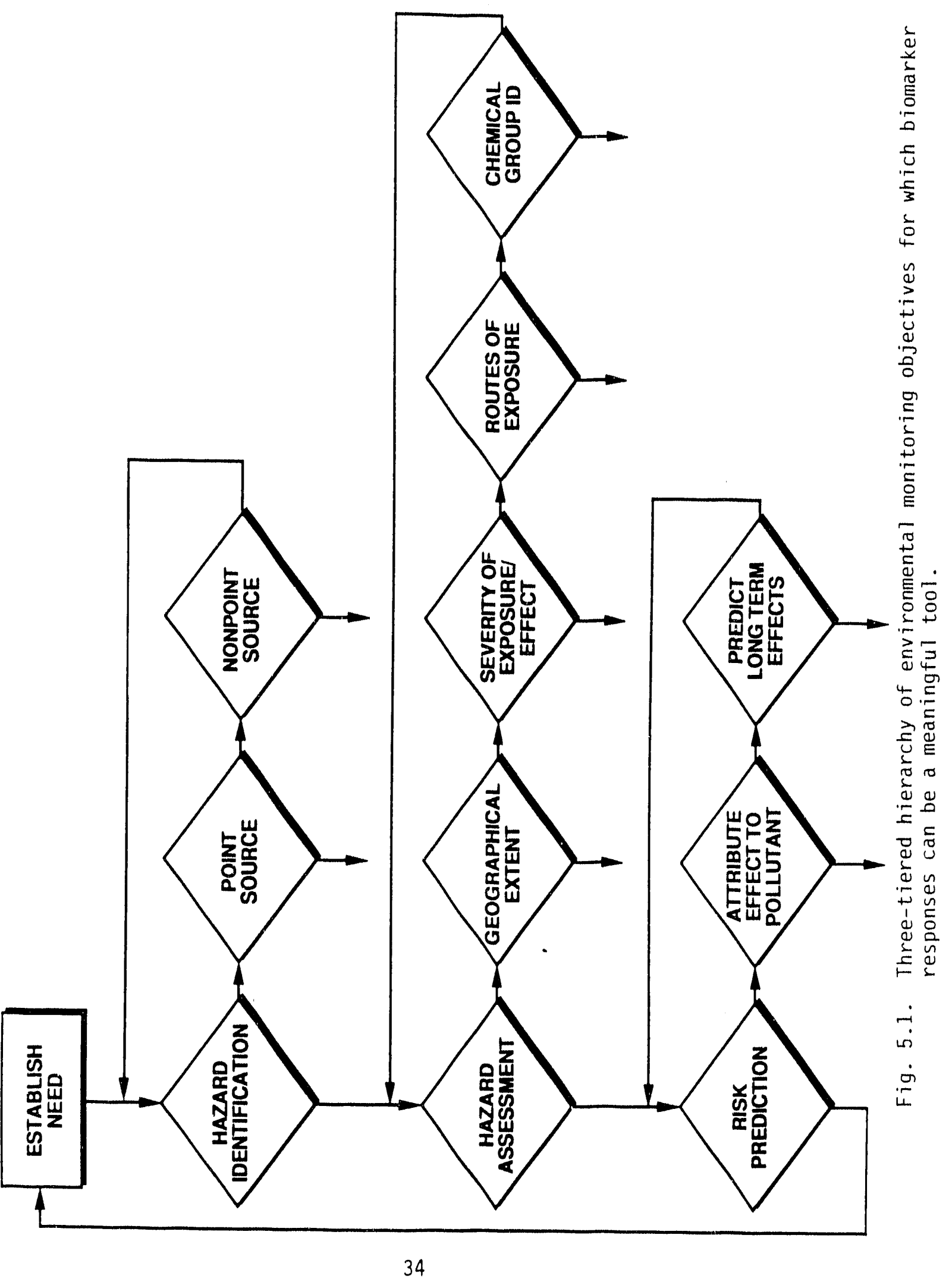


Several questions can arise once a chemical is known to be present or once exposure is known to have occurred. Exposure as used here means that a xenobiotic has entered an organism and is detectable by biomarker responses beyond that normally seen in homeostasis. Questions of particular concern include

- What is the geographical extent of the exposure?

- What is the severity of the exposure/effect?

- Are there trends in exposure over time?

- What are the routes of exposure (water, soil, sediment, or food chain)?

- What group of chemicals are involved?

Research at this level of the biomarker hierarchy differs from that discussed under Hazard Identification in that biomarkers that are more specific in response are often chosen for analysis. At this level the emphasis is less on screening-level surveys of potential problems and more on improved definition and description of identified areas of concern. The results of an Hazard Identification study may have indicated that exposure has occurred; now the question is, What chemical groups are involved and/or what is the geographical extent of the exposure? Similarly, a general survey of exposure (Hazard Identification) in the Chesapeake Bay may have indicated exposure; now the question is, What is the route of exposure? In addition, trends in exposure over time can be tracked through changes in biomarker responses. This application can be used to monitor remedial efforts at toxic waste sites or to track regional or global fluctuations in environmental pollution. It is difficult to predict the questions that may arise once exposure is known to have occurred or specific chemicals are known to be present. Those responsible for answering the questions will have to prioritize the research and determine the degree of detail needed to respond to specific questions.

\subsubsection{RISK PREDICTION}

- Third tier in the hierarchy of biomarker uses

- Linking biomarker responses to effects at the population or community level

- Linking biomarker responses in sentinel species to human epidemiology 
This is the ultimate long-range goal of biomarker-based biomonitoring. Given a continuing level of chemical exposure and detecting a suite of blomarker responses, is it possible to predict communitylevel effects (e.g., reduction in species diversity, changes in predator/prey relationships, trophic level changes, or increased incidence of disease)? The basic questions that arise at this level of the hierarchy of biomarker uses are

- What ecological effects (or possible effects in humans) will be observed if the current level of exposure continues?

- Are the observed community-level effects attributable to exposure from a specific xenobiotic source?

These two questions, although related, deal with separate issues. To the regulator, it is important to identify the source of effects so that specific remedial actions may be taken. This may be very difficult in multiple-discharge situations where individual effluents produce no effects but where the synergistic action of several effluents do produce effects. An example would be a bay or river receiving multiple municipal and/or industrial effluents. To an environmental scientist or to the human population living in an exposure zone, the prediction of potential long-term effects may be a more pressing question.

\subsection{GENERAL ELEMENTS OF A BIOMARKER-BASED BIOMONITORING STUDY}

Regardless of the specific objectives or motivation for a monitoring study, many elements of the study design remain constant. Figure 5.2 illustrates the principal elements of the design and implementation of a biomonitoring study in a logical pathway. In the following pages, major issues within each element are outlined, along with a checklist of specific considerations to be addressed in arriving at a final study design. Although the general elements, issues, and considerations are largely unchanged by the study objectives, the answers to the checklist queries, and the weight or importance to be assigned to that issue in the final study, design will vary with specific objectives, resources available, types and sources of pollution, and accumulated field experience. 


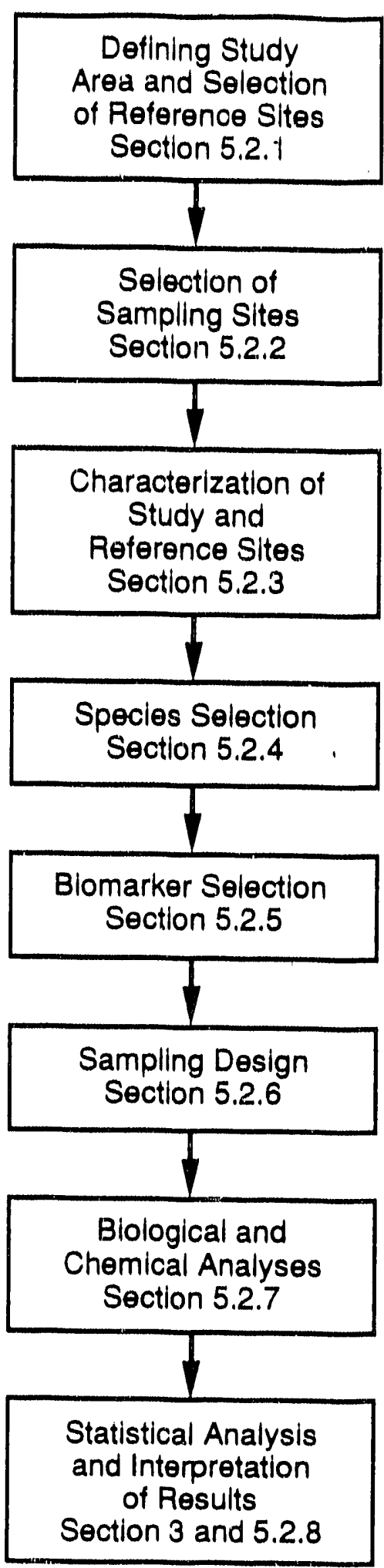

Fig. 5.2. Principal elements of a biomarker-based biomonitoring research program. 


\subsubsection{Defining Study Area and Selecting Reference Sites}

- Boundaries

- Area, length, and/or width

- Select ecologically similar reference sites

\section{- Boundaries}

- Have the limits of the study area been concisely defined?

- Are the boundaries readily identifiable?

- Are there activities in bordering areas that may influence the results of this study?

- Are there any reasons why adjoining property owners should be notified of research activities?

- If the study is designed to validate a specific biomarker, is the chemical history of the selected study area well characterized?

- Area, length, and/or width

- Is the study area of reasonable size for the planned research and resources available?

- Select several ecologically similar reference sites

- Are there any influences that may effect the interpretation of results obtained from the reference sites?

- Are the reference sites similar enough to the study site so that valid comparisons can be made?

Ideally reference sites should be identical in all respects to study sites except that they are free of any contamination. Because this is an impossibility, efforts should be made to find reference sites that are similar in geographic location, habitat, size, topography, etc., to the study site. 


\subsubsection{Selection of Sampling Sites}

- Define

- Habitat types

- Access

- Compatibility with sampling methods

- Relationship with source of contamination

- Statistical considerations

- Define

- Have the boundaries of the sampling sites been established?

- Are the boundaries easily recognizable?

\section{- Habitat types}

- Are the habitat types selected reasonable based on knowledge of chemicals present and suspected routes of uptake?

- Are the habitat types of study and reference sites similar?

- Have all habitat types been considered?

- Are well-established sampling techniques available for the habitat type selected?

- Are there any anticipated changes (e.g., logging, dam building, or agricultural) in study or reference sites?

\section{- Access}

- Is there adequate access available to the sampling sites?

- Are there any anticipated changes in access?

- Has trespass been considered, and do property owners need to be contacted?

- Will access be available during all seasons and all years (if needed)?

\section{- Compatibility with sampling methods}

- Are the sites selected compatible with available sampling methods?

- Have alternative sampling methods been considered? 
- Relationship with source of contamination

- Are sampling sites located sufficiently close to point sources?

- Have all possible contaminated sites been considered?

- If a gradient is being determined, are the sampling sites located at reasonable distances and in a reasonable pattern?

\section{- Statistical considerations}

- Are number and location of selected sites compatible with anticipated statistical analysis?

\subsubsection{Characterization of Study and Reference Sites}

- Species present/absent and relative abundance

- Population demographic information

- Water, sediment, and soil chemistry

- Species presence/absence and relative abundance

- Will sampling have to be done to determine what species are present or is that information already available?

- What species are present in sufficient numbers for sampling?

- Are the same species present at study and reference sites in approximately the same abundance?

- What species are present in sufficient numbers for sampling?

- Are vertebrates, invertebrates, and/or plants of interest?

\section{- Population demographic information}

- What is the sex and age distributions of the populations present in sufficient numbers for sampling?

- Do the demographics support the selection of the reference sites as representative of undisturbed habitats? 
- Water, sediment, and soil chemistry

- Is water, sediment, and/or soil chemistry data already available for the sites?

- Is there sufficlent chemical data available to warrant a more comprehensive chemical analysis of water, soll, and/or sediment?

- Is it necessary to acquire new/more complete data on environmental concentrations of contaminants (e.g., to improve site selection or establish gradient of pollution)?

\subsubsection{Specics Selection}

- Vertebrates, invertebrates, and/or plants

- Sensitivity to chemicals

- Home range/mobility

- Abundance, sex, and age

- Habitat and trophic level

- Metabolic capabilities/toxicological mechanisms

- Vertebrates, invertebrates, and/or plants

- Are vertebrates, invertebrates, and plants all available for sampling?

- Is there a preference for one group of organisms over another?

- Have effects been observed in any group of organisms?

- Has consideration been given to introducing species (free living or caged)?

- Is one group of organisms known or likely to have a greater degree of exposure?

- Are there sufficient expertise and resources to allow analysis of more than one group of organisms?

- Are food web effects or routes of exposure important in this study?

- Sensitivity to chemicals

- Is there knowledge of or does it appear that one species or group of organisms is more sensitive to chemicals known or suspected to be present?

- Is there a particular biomarker response of interest for which a particular group of organisms is required (e.g., mammalian MFO)? 


\section{- Home range/mobility}

- Is the home range of the species being considered within the boundary of the study site? Match home range to size of study area and degree of geographic resolution desired for study.

- Is a wide-ranging species (more opportunity for exposure) desirable?

Broad ranges provide integration of exposure over wide geographical areas but at the expense of loss in geographical resolution.

- Is information needed on small, very specific areas?

Sessile sentinels (e.g., plants and clams) offer geographical resolution but may not be representative of overall exposure.

- Abundance, sex, and age

- Are there sufficient numbers to sample without affecting the population?

- Is a particular sex or age more susceptible to chemicals that are present?

- Will the blomarker(s) selected for analysis change with sex or age?

- Will changes in reproductive cycle affect the results of this study?

- If a specific sex or age group is selected, are they available in sufficient numbers for sampling? Ideally, individuals selected for study should come from the same sex and age class and have similar reproductive and nutritional status.

- Are the species being considered of sufficient size to provide large enough samples for all anticipated analysis or will pooling be necessary?

\section{- Habitat and trophic level}

- Would selection of a species from a higher trophic level be useful in integrating exposure over a broad range of potential exposure pathways?

- Are trophic level effects of interest?

- Is there sufficient information on trophic pathways?

- Should organisms be selected from specific habitats to better define the significance of different routes of exposure (e.g., soil-associated vs water column exposure)?

\section{- Metabolic capabilities/toxicological mechanisms}

- Are xenobiotic metabolizing capabilities of the species known?

- Does the species have the capability of activating compounds to toxic intermediates and/or detoxifying and eliminating contaminants, and are the capabilities representative of those of other species of concern? 


\subsubsection{Biomarker Selection}

- General vs specific response

- Species sensitivity

- Resources available

- General us specific responso

- Are biomarkers that respond to a variety of chemicals (exposures) desirable, or are biomarkers that respond io a specific group of chemicals desirable?

- Is there information available on specific chemicals that might be present for which specific biomarker analyses would be appropriate?

- Has a suite of biomarkers representing both general and specific responses, fast vs slow, or transient vs persistent been considered?

\section{- Species sensitivity}

- Are there specles present that are known to be particularly sensitive to chemicals that may be present for which specific biomarkers have been previously analyzed?

\section{- Resources available}

- What resources are available for analysis of biomarkers?

- Are resources available for analysis of a suite of biomarkers in-house or can analyses be performed by qualified specialists at universities, etc.?

- Have other agencies been queried for available resources? 


\subsubsection{Sampling Design}

- Season/time

- Sample size

- $\mathrm{QA} / \mathrm{QC}$

- Sampling methods

- Resources available

\section{- Scason/time}

- Have the effects of changes in season on species abundance and biomarker response been considered?

It is critical that all sites (study and reference) be sampled in the same season and, preferably, at or about the same time.

- Will seasonal changes have an influence on sampling methods?

- Will seasonal changes affect access?

- Has consideration been given to sampling for more than one season and for more than one year?

- Have behavioral changes in species been considered?

\section{- Sample sizes}

- Are sample sizes going to be sufficient to allow comparisons between sex and age groups?

- Has a statistician been consulted regarding sample sizes?

\section{- QAVC}

- How and by whom will samples be collected, stored, and transported?

- Has an adequate chain of custody been established?

- Have sufficient measures been taken to accommodate unforeseen happenings (delayed transportation, shipping, sickness, etc.)?

- Do laboratories doing assays have approved QA/QC procedures? 


\section{- Sampling method}

- Will standard and tested sampling methods be used?

- Are field crews adequately trained in sampling methods to be used (species collection and sample collection and preservation)?

- Will similar sampling methods be used at all collection sites and will similar efforts be devoted to collections in study and reference areas?

- Resources available

- Is the sampling design practical, given the resources available (e.g., time, money, personnel, and equipment)?

\subsubsection{Biological and Chemical Analyses}

- Biomarker analyses

- Tissue chemical analysis

- General health status of organisms

\section{- Biomarker analyses}

- Are analyses being done by experienced personnel with adequate QA/QC documentation?

- Are analyses being compared among laboratories?

- Tissue chemical analysis

- Are tissue samples analyzed by approved and consistent methods?

- If specific chemicals are unknown, are tissues being screened for a range of organic and inorganic contaminants?

\section{- General health status of organisms}

- Are all anomalies being recorded?

Is the incidence of disease and the number of observable lesions being recorded?

- Are differences in sex and age ratios being recorded? 


\subsubsection{Statistical Analysis and Interpretation of Results}

- Statistical analysis

- Interpretation

- Statistical analysis

- Has a statistician familiar with analysis of biological data been consulied?

- Has the data been checked for normality, and will parametric or nonparametric analysis be used?

- Have multivariate analyses been considered?

- Has the data been plotted and does the anticipated analysis and results make sense?

Counterintuitive results may indicate interaction of ecological, physiological, or toxicological variables on biomarker response. More research may be indicated to elucidate these interactions (see Sect. 5.4).

- Have descriptive statistics been considered (e.g., percentage change in a variable between study and reference sites or for multivariate responses or Mahalanobis generalized distance between sites)?

\section{- Interpretation}

- Has all the information needed for interpretation been collected and analyzed?

- Are the results consistent with anticipated outcome?

- How do the results compare with previous studies?

\subsection{TAILORING STUDY DESIGN FOR SPECIFIC OBJECTIVES}

This section focuses on applications that are sperific to particular objectives. The objective and applications are shown, and characteristics that tend to separate one application from another are listed (Figs. 5.3, 5.4, and 5.5). Although the characteristics listed can be used to separate applications, they are not necessarily unique to the particular application and should be considered in a general descriptive context. 


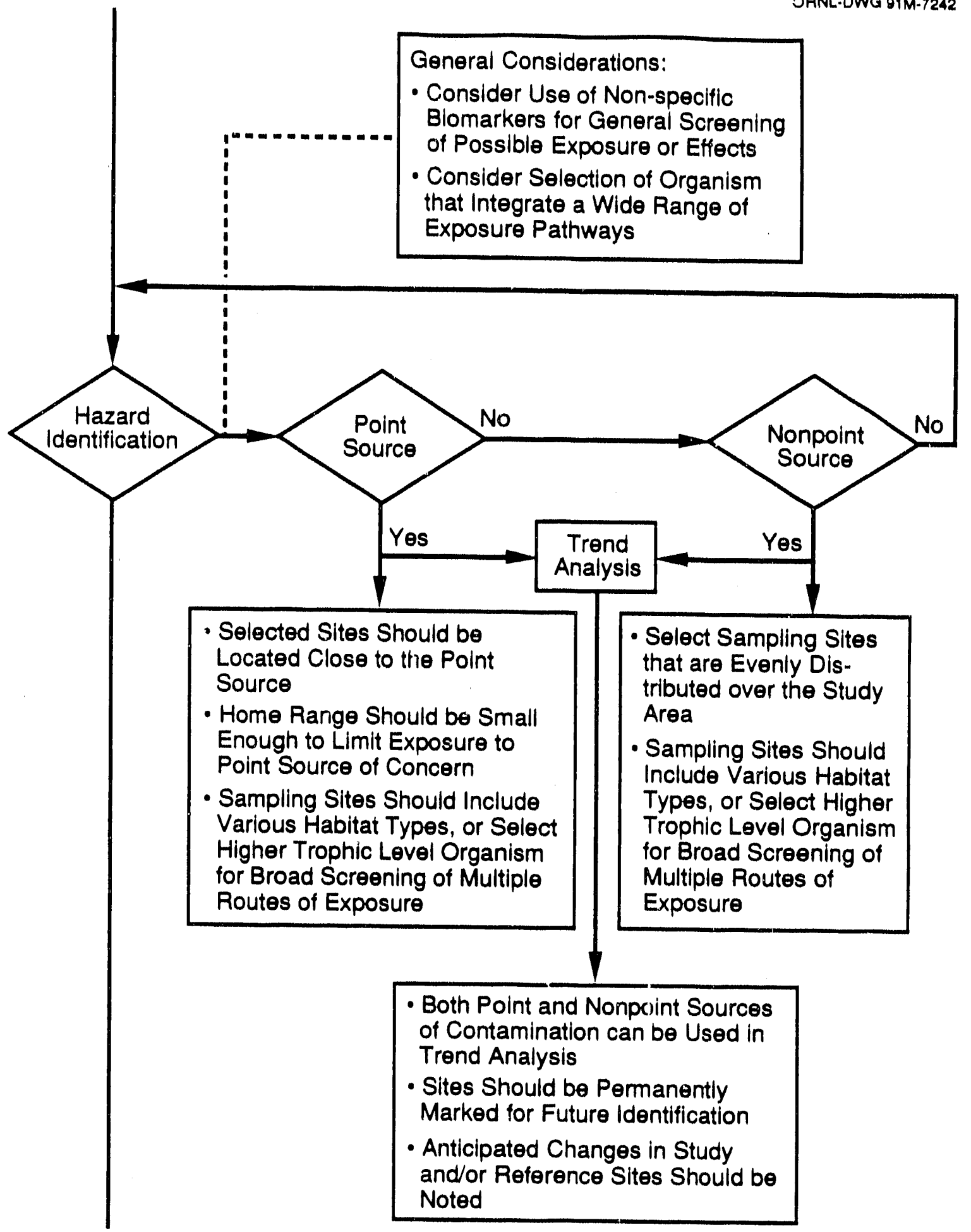

Fig. 5.3. Characteristics that can be associated with the Hazard Identification tier of the Hierarchy of Environmental Monitoring Objectives. 


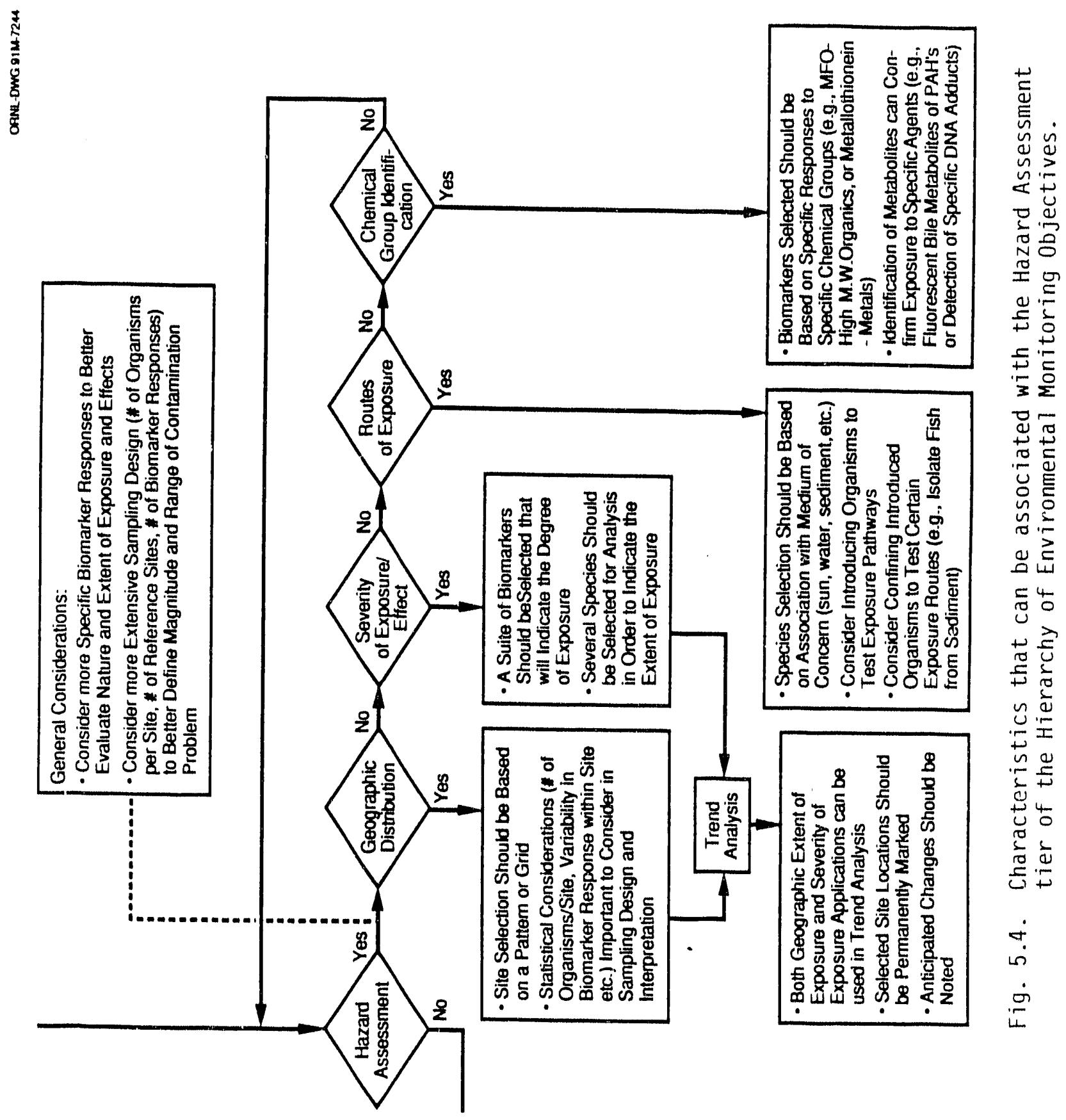




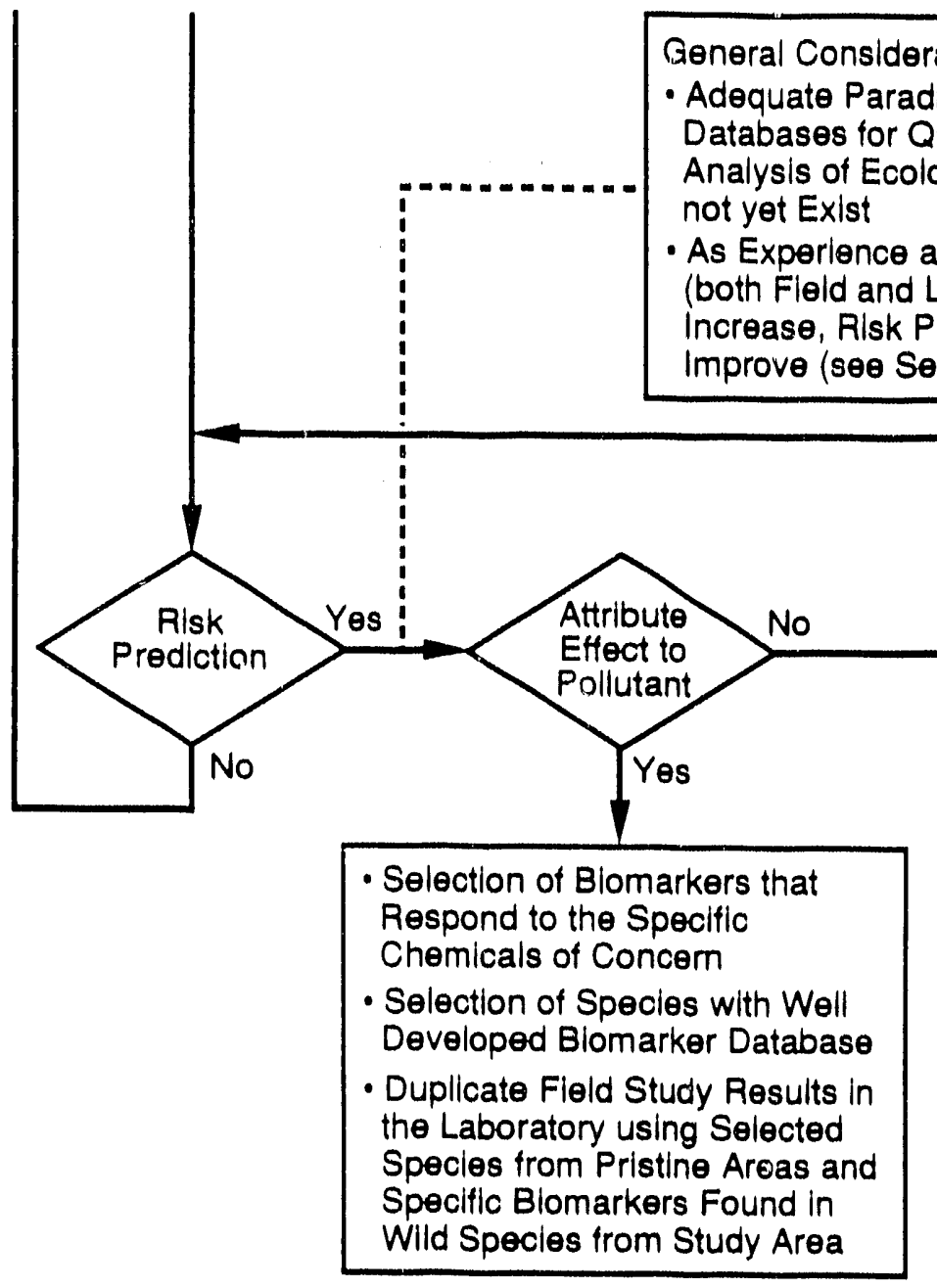

General Considerations:

- Adequate Paradigms and

Databases for Quantative Rlsk

Analysis of Ecological Effects do

Risk Prediction will

improve (se日 Sections 5.4 and 7 )

Selection of Blomarkers that

Respond to the Specifio

Chemicals of Concerm

Selection of Species with Well

Database

Specific Blomarkers Found in

Wild Species from Study Area

- Selection of Species with Well
Developed Blomarker Database
- Selection of Specific Blomarkers
that have been Shown to be
Indicative of an Impending
Disease State

Fig. 5.5. Characteristics that can be associated with the Risk Prediction tier of the Hierarchy of Environmental Monitoring Objectives. 


\subsection{RESEARCH COMPONENTS IN DEVELOPMENT OF BIOMARKERS: LABORATORY AND FIELD STUDIES}

This section builds off the previous pathway that presented the general elements of a biomarkerbased biomonitoring study. The pathways presented here are designed for use in the biomarker research and evaluation strategy needed to validate specific biomarkers before they can he used in environmental monitoring (Fig. 5.6). This conceptual pathway focuses on designing parallel laboratory and field research to

(1) improve basic understanding of biomarker responses;

(2) elucidate the role of environmental and toxicological stresses on biomarker responses; and

(3) confirm relationships between exposure, biomarker responses, and long-term adverse effects to individuals and populations.

Many fundamental research questions can be probed by straightforward experimental design. For example, dose-response studies or questions related to the effect of single variables on specific biomarker responses (e.g., effect of reproductive status on contaminant-related induction of MFO activity) can be designed and executed based on classic approaches in the toxicological literature. However, development of a biomarker-based biomonitoring capability will require a close integration of controlled laboratory studies in conjunction with ongoing field studies. It is the purpose of this section to identify key components of an integrated laboratory-field program. As in Sect. 5.2, the new segments of the pathway (highlighted segments) are followed by a number of queries that address important considerations pertinent to the particular segment listed.

\subsubsection{Define Experimental Objective}

- Clear statement of experimental objective

- Relationship between exposure and biomarker responses

- Relationship between biomarker responses and health/ecological effects 


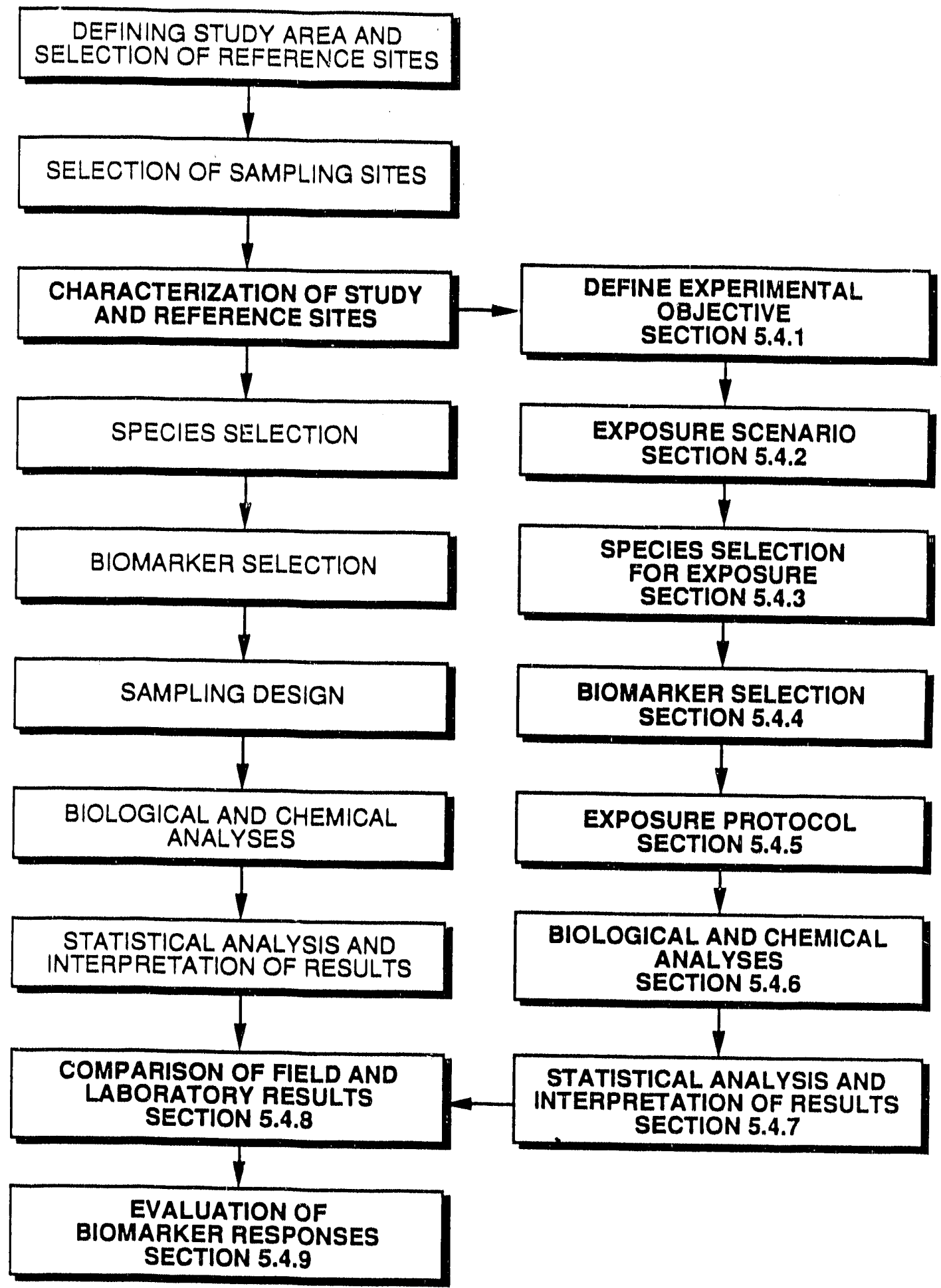

Fig. 5.6. Elements of a biomarker-based biomonitoring research program with associated experimental phase. 


\section{- Clear statement of experimental objective}

- The goal is to provide information that will aid in the understanding, interpretation, and definition of biomarker responses observed in field studies of wild exposed species. This is accomplished through exposure of individuals of the same species (or another appropriate species) collected from pristine environments (or possibly from the laboratory) to contaminants found at the study site.

- The objective is to support and improve interpretation and understanding of field responses by defining and isolating what are thought to be key controlling variables or interactions in a laboratory setting.

- The environmental focus may be on understanding and evaluating a specific biomarker or examining quantitative and qualitative responses of a suite of biomarkers in response to a given exposure scenario.

- Relationship between exposure and biomarker responses

- Test dose-response relationships between exposure and biomarker, especially with respect to effect of potentially confounding variables

- interactions with other chemical contaminants (individual compounds, rationalized mixtures of chemicals, or complex mixtures representing effluents or contaminated media)

- exposure media/routes of exposure (water, air, or ingestion)

Compound pharmacodynamics and ultimate effect may depend on mode of entry into organism.

- physiological variables (sex, age, reproductive status, or species-specific metabolic capabilities or sensitivities)

- ecological stressors (temperature, food, availability, or exposure to disease)

- acute or chronic exposure

- Relationship between biomarker response and health/ecological EFFECTS

- Establish relationships between biomarkers and long-term consequences of exposure.

Design experiments so that the pattern of biomarker response will indicate if organisms are able to compensate for exposure, are forced to spend additional energy to repair contaminant-induced damage, or are unable to effectively repair damage and, as a result, enter a diseased state (see Fig. 2.1). 


\subsubsection{Exposure Scenario}

- FOCUS EXPERIMENTAL DESIGN

- SELECTED CHEMICALS OR MIXTURES

\section{- Focus experimental design}

- Is the emphasis of study on biomarker response to controlled exposures?

Relatively short-term exposures may be adequate.

- Are long-term health or ecological consequences of primary interest?

These require more-complex and "fail-safe" exposure facilities, larger number of animals, and considerably greater resources.

- Is emphasis on specific effects of a contaminant (or mixture) or an effect of physiological or ecological factors on biomarker response?

Can the variable of interest be adequately simulated and controlled over exposure period?

- Is emphasis on an individual species, or should design include multiple populations in a mesocosm facility?

- If reproductive endpoints are of interest, is it necessary to test viability and development of offspring?

- How many organisms are needed and what experimental controls and sampling design are required for appropriate statistical analysis?

Consult a statistician BEFORE experimental design is finalized. 


\section{- Selected chemicals or mixtures}

- Are specific chemicals of concern and should selected species be exposed to a mixture of these chemicals in a laboratory setting?

- Should species be exposed to single chemicals and/or mixtures of chemicals?

If field exposure is to multiply chemicals, it may be possible to expose oryanisms collected from pristine environments to single chemicals and various combination of chemicals to aid in identification of specific chemicals that are responsible for adverse effects (biomarker responses).

- Is it possible to expose selected species at the study and reference sites to maintain realistic environmental conditions?

- Should there be joint exposure to specific chemicals, mixtures, and/or effluent, water, soil, or sediment?

- Are there other exposure scenarios that are practical and that would help to maintain as much similarity between the controlled experiments and what is happening (environmental influences) at the study and reference sites?

\subsubsection{Species Selection:}

- Laboratory vs wild species

- Sensitivity to chemicals

- Habitat and trophic level

- Sex and age

\section{- Laboratory vs wild species}

- Would exposure studies using wild species (same species selected for analysis from the study site) be practical?

Collecting wild species from a "pristine" area and exposing them to study site effluent, water, etc., may provide more appropriate data to compare with data collected from that species collected at the study site.

- If laboratory species are selected for exposure studies, do they have similar metabolic capabilities as the species selected for analysis from the study site?

Because field and laboratory studies will be conducted concurrently during the biomarker validation phase, researchers should ensure that species selected for laboratory studies are comparable in biomarker response to those being analyzed from the field. 
- Is there a data base of information on blomarkers in the species selected?

- What would be the added value gained by looking at more than one species?

\section{- Sensitivity to chemicals}

- Is the species selected known to be sensitive to the chemicals present?

- If information is not available on species sensitivity or the specific chemicals are not known, has consideration been given to selection of a species for which a biomarker data base is available?

Selection of a species for which a biomarker data base is available and also a wild species present at the study site may provide a good combination of results for evaluating biomarker responses to the specific exposure used.

- Route of exposure: Habitat and trophic level

- Would selection of a species from a higher trophic level be useful in integrating exposure over a broad range of potential exposure pathways?

- Are trophic level effects of interest?

- Is there sufficient information on trophic pathways?

- Should organisms be selected from specific habitats to better define the significance of different routes of exposure (e.g., soil-associated vs water-column exposure)?

- Sex and age

- Have response differences based on sex and age been considered?

- Are thère reproductive effects that confound interpretation of results?

- Is it possible that the response of the selected species will vary with seasonal changes, and should samples be collected during a different season or over several seasons? 


\subsubsection{Biomarker Selection}

- Sulte of biomarkers

- Test new biomarkers

- Similarity between field and laboratory study

- Archive tissue

\section{- Suite of biomarkers}

- Should the study focus on further understanding of one biomarker or on time course and interaction of a suite of biomarkers?

- Does the suite of biomarkers selected for analysis include biomarkers of both the general- and specific-response type?

In the validation phase of biomarker research, it is important that a diverse suite of biomarkers be selected for analysis.

- Have field personnel been instructed on the proper methods to use in collecting and preserving tissues needed for biomarker analysis?

- Are resources available and is the time schedule practical for exposure and collection of the biomarkers being considered?

Exposure periods for different biomarkers may differ and consideration should be given to sample sizes needed for collection of tissues over different time periods.

\section{- Test new biomarkers}

- Has consideration been given to the testing of promising new biomarkers in controlled studies to develop, test, and validate these responses for future applications?

It may be valuable and more efficient to include testing of new biomarkers in studies using a suite of more established and well-characterized biomarkers.

\section{- Similarity between field and laboratory studies}

- Should a specific aspect of field exposure be isolated for laboratory study (e.g., specific chemicals or mixtures of chemicals, routes of exposure, and/or ecological, physiological, or toxicological concern)? 
For example, reproductive status, food stress, disease condition, etc., are potentially critical variables that can be isolated to define their impact on the response of single biomarkers or on patterns of multiple biomarker responses.

- Will the same blomarkers be analyzed in field and laboratory samples?

Developing well-documented controlled-exposure data bases on the time course and quantitative and qualitative pattern of biomarker response to specific chemicals, to rationalized mixtures, or to whole effluents/sediments is critical to validation and interpretation of biomarker responses.

- Archive tissues

- Has consideration been given to archiving tissues (from both laboratory and field species) for later analysis?

It is possible to store tissues so that analysis for different biomarkers can be performed as interest develops or as additional methods or techniques become available.

- Is there ample storage space, and what is the proper storage method?

- Do backup and fail-safe systems ensure sample integrity?

- Will alarm systems document failure of storage system?

- Can the tissues be processed prior to storage?

Depending on anticipated future needs, tissue samples may go through some initial processing (e.g., isolation of microsomes and DNA) prior to storage. This may reduce the space needed and better preserve the integrity of the collected samples.

\subsubsection{Exposure Protocol}

- Realistic exposure

- Sample size

\section{- Realistic exposure}

- Is the concentration that the organisms are exposed to similar to that found in the study area?

- Do there appear to be long term health effects, and will exposure time be sufficient to allow observation of long-term effects? 
- Are other exposure conditions (e.g., temperature, light/dark cycle) as similar as possible to the environmental conditions found at the fleld sites?

- Is it possible to simulate other sources of stress (e.g., predator/prey) to which the organisms would be subjected in the wild?

- Sample size

- Is the sample size sufficient to allow samples to be collected at different times during the course of the experiment?

- If various sex and age groups are being sampled, are there sufficient numbers of each sex and age group?

- Have the requirements for statistical analysis been considered?

\subsubsection{Biological and Chemical Analysis}

- Biomarker analysis

- Tissue chemical analysis

- General health status of organism

\section{- Biomarker analysis}

- Are the same biomarkers being analyzed in laboratory exposures as in field samples?

- Are analyses being done by experienced personnel?

- Are analyses being compared among laboratories?

\section{- Tissue chemical analysis}

- Are chemical concentrations being determined in the same tissues analyzed in field samples?

- Are tissue samples analyzed by approved methods?

- If specific chemicals are unknown, are tissues being screened for a range of contaminants? 


\section{- General health status of organism}

- Are all anomalies being recorded?

- Is the incidence of disease and the number of observable lesions being recorded?

- Are the individuals performing necropsies adequately trained?

- Are sex and age ratios being recorded?

- Are body and organ measurements (e.g., weight and length) being recorded?

Differences in body and organ measurements by age and/or sex group may je an important indicator of effects on normal physical and reproductive development.

\subsubsection{Statistical Analysis and Interpretation of Results}

- Statistical analysis

- Interpretation

\section{- Statistical analysis}

- Have the results been compared using standard statistical methods (see Sect. 3 for discussion)?

Use of hypotheses-testing statistics may be appropriate for properly designed laboratory studies.

- Has a statistician familiar with analysis of biological data been consulted?

- Has the data been checked for normality, and will parametric or nonparametric analysis be used?

- Have multivariate analyses been considered?

- Has the data been plotted, and do the anticipated analysis and results make sense?

- Have descriptive statistics been considered (e.g., percentage change in a variable between study and reference sites)?

\section{- Interpretation}

- Has all the information needed for interpretation been collected and analyzed?

- Are the results consistent with anticipated outcomes? 
- How do the results compare with previous studies?

- Do the results make sense based on current toxicological knowledge?

\subsubsection{Comparison of Field and Laboratory Results}

- Statistical comparison

\section{- Statistical comparison}

- Have the field and laboratory results been compared using standard statistical methods (see Sect. 3 for discussion)?

- Are the results realistic, and do they make sense?

- Have possible sources of error or differences between laboratory and field conditions been considered?

- Have the results of the laboratory study provided the information needed to understand, interpret, and/or better define the selected biomarkers?

\subsubsection{Evaluation of Biomarker Responses}

- Comparison with previous studies

- Additional studies

- Comparison with previous studies

- Are the results similar to the results obtained from previous studies?

- Have differences do to species, sex, age, and season been considered in comparisons with previous studies?

- Do the results of this study make sense compared with the results of previous studies? 
- Additional studies

- Are additional experiments needed before biomarker responses can be interpreted?

- Should the experiments be repeated or additional samples collected from the field before this study is terminated?

- Are resources available for additional analysis or collection?

- Are the biomarker responses good indicators of exposure and effects?

- Can any biomarker or groups of biomarkers be used to indicate if the organism has moved from compensatory response to repair response (see Sect. 2 for discussion)?

- Are there single biomarkers or groups of biomarkers that can be used to predict future events (e.g., disease or death)?

- How could the experiments and field sampling have been improved? 


\section{SELECTION AND PRIORITIZATION}

\subsection{SELECTION}

It is obvious, from both logistical and economical points of consideration, that a biological monitoring program can not include a large number of biomarkers. Guidelines outlined in Sect. 5.2.5 of this document will dictate to a great extent the type and number considered suitable, but even at this point in the selection process, the list of potential biomarkers might be too large. If several general attributes and characteristics of biomarkers are recognized and considered, then selection can be finetuned without diminishing effectiveness. Factors to be considered are

Response-It should not be anticipated a priori that exposure of an organism to contaminants in its environment will elicit a biological response or that responses will be observed through different levels of biological organization. Where possible, biomarker selection should be based on the known toxicological mechanism of action of the contaminants present, thus maximizing the probability of observing a biological response(s).

Time frame-Some biomarkers are measurable early (hours) after exposure and are observable at the biochemical/molecular level of biological organization. These early responses may exhibit a temporal existence. Others appear much later (years) and are seen at higher levels of organization. Implicit in the concept of biomarker use is the potential for correlating responses among various levels of biological organization.

Variability-Sources of variability that may influence the measurement of a biological response generally fall into two categories: those inherent to the laboratory method, procedure, or assay selected and those intrinsic to the species being sampled. Sample collection, preparation, and storage as well as reagent purity and instrument selection and calibration are examples of the first category. They are more easily controlled through adherence to quality assurance and quality control policies. Age, sex, and disease state of the organism being sampled or environmental stresses such as climate or food availability are examples of factors that contribute variability in the second category. The effects of these latter factors on the 
biomarker assay is difficult to predict; however, they can be documented and accounted for by establishing baseline data from appropriate noncontaminated or reference sites.

Limitations-Specific limitations and restrictions apply to the use and interpretation of many of the biomarkers, and these factors should be verified by consulting the current scientific literature.

\subsection{PRIORITIZATION}

The prioritization process involves the selection of biomarkers that are appropriate to the objectives of a given biological monitoring program. The suitability of a particular biomarker should be judged on the anticipated probability of obtaining information that will document either exposure, status of cellular compensatory mechanisms, or potential for harm. An in-depth survey of biomarkers was recently conducted (Huggett et al. 1991). From such a survey it is possible to identify a small subset of biomarkers with which to examine and evaluate both specific or general types of contamination problem. The identification of this subset and its supplementation represents the first step in the prioritization process.

For example, consider the following two environmental contamination problems. In one instance the concern is for toxic metals; in the second, the presence of genotoxic agents. Shugart et al. (1989) has proposed a suite of biomarkers to monitor for anticipated biological responses with regard to the former problem:

xenobiotic metabolism

immune response

DNA integrity

metal-binding proteins

porphyrin biosynthesis 
In the latter problem the list of biomarkers described in Table 7.1 would constitute a reasonable core, namely,

DNA damage (both specific and general)

DNA repair

xenobiotic metabolism

chromosomal aberrations

In both instances the suite of biomarkers chosen addresses the identified concern by including biomarkers that could provide information on exposure (whether it has occurred), on the status of compensatory mechanisms (has the organism's capacity to maintain homeostasis been compromised), and indications of harm (have irreversible events occurred). As with any suite of biomarkers, the interpretation of the observed response must be tempered by the current state of our scientific knowledge (i.e., specific limitations and restrictions). Some responses will be definitive indicators of exposure and even predictive of long-term adverse effects; whereas other responses will only be a signal of a potential problem.

The collection of data from an appropriate reference or control site cannot be overemphasized. Once adequate baseline information concerning the suite of biomarkers is established for a species or set of species within a defined area, one would be able to sample that species or species set from selected sites and compare the results with established baselines. We hope that such comparisons will help define the influence of or contribution of intrinsic factors that contribute to variability.

Although biomarkers cannot at this time be employed to predict effects at higher levels of biological organization (i.e., population-, community-, and ecosystem-level responses), it is important to note that appropriate use of these biomarkers may provide very sensitive, early warning signals of incipient ecological damage. 


\section{BIOMARKERS AS ENDPOINTS IN TOXICITY TESTING}

\subsection{INTRODUCTION}

Toxicity tests may be either short-term or long-term. In the short-term test systems, for example, early life stages of a species are examined for the effect(s) of exposure to toxic substances and the endpoints of concern are survival, growth, and development. In the longer-term tests, latent events such as neoplasia and reproductive capacity of survivors are measured.

Perhaps the single greatest concern related to the release of anthropogenic pollutants into the environment is the potential for exposure to carcinogenic chemicals. However, the development of neoplasia, tumors, or other pathological responses of carcinogenesis is a slow process that is dependent on numerous unknown and ill-defined factors. Therefore, these endpoints are not amenable to examination in short-term toxicity testing but require expensive long-term exposure. The predictive power of toxicity testing in this area could be greatly increased if a suite of rapidly responding biomarkers for genotoxicity were included as an additional analytic component.

\subsection{CASE IN POINT: GENOTOXICITY TESTING IN FISH}

The primary endpoint for most small fish carcinogenesis studies on small fish is the histopathogenic identification of a neoplastic lesion. Such lesions occur long after exposure and are found mainly in the liver, although other tissues in which lesions have been observed include nervous tissue, kidney, mesenchymal tissue, and skeletal and smooth muscle. The histogenesis of liver neoplasms in fish is similar to that in rodents. Following exposure to chemical carcinogens, preneoplastic lesions appear as eosinophilic foci and basophilic foci, followed by tumor formation (Hawkins et al. 1988). This is identical to the process that appears to occur in rodents and other mammals (Farber 1987).

The most promising biomarkers for detecting early exposure to carcinogenic chemicals are those that measure the interaction of these compounds with the cellular macromolecule. Thus, by definition, they are biomarkers of genotoxicity. The biological endpoint assumes significance because those interactions that cause damage to DNA may be the critical events leading eventually to adverse effects (neoplasia) in the exposed organism (Harvey 1982). 
Many chemical carcinogens and mutagens have the capacity to cause various types of DNA damage as a result of their metabolism (i.e., production of highly reactive metabolites, free radicals, etc.). Damage usually occurs shortly after exposure and cause alterations to DNA such as modifted bases and adducts, some of which result in the formation of alkali-labile apurinic sites and some of which are unstable and cause strand breaks (Shugart 1990). Indirectly, the fidelity of postreplicative modification of DNA (such as minor nucleotide composition) can be affected by genotoxic agents (Jones 1987; Shugart 1990). Each type of damage to the integrity of the DNA, if left uncorrected, could trigger a sequence of cellular events that culminates in the appearance of an overt malignancy. These various types of early damage to DNA, along with an appraisal of the organism capability to maintain the integrity of it's DNA (repair), constitute biomarkers of genotoxicity. The detection of these initial events in the carcinogenic or mutagenic process provides the basis for determining whether exposure to genotoxic agents has occurred.

\subsection{APPLICATION}

In toxicity testing an evaluation is made on the basis of the suspected presence of some deleterious chemical(s) or contaminant(s), for example, water quality testing (U.S. Environmental Protection Agency), sediment toxicity testing (U.S. Army Corp of Engineers), and animal bioassys for carcinogenesis (National Institutes of Health). Biomarkers of genotoxicity would be useful in these tests because they could signal the presence of an unsuspected genotoxic chemical that would not normally be detected with the conventional endpoints of the test used as in the first two examples (i.e., measurement of strand breaks in DNA or the detection of the induction of DNA repair in the fathead larvae at the termination of a standard minichronic toxicity test). Alternatively, these biomarkers of genotoxicity could be short-term predictors of potential long-term effects, as in the tests for carcinogenesis (i.e., correlation of early genotoxic responses with subsequent latent events such as the occurrence of aneuploidy or transformation of initiated cells). An often overlooked ancillary benefit derived from the application of biomarkers is the savings in cost, especially as an alternative to tests involving expensive animal models of carcinogenesis and mutagenesis (i.e., Japanese medaka or rodent).

A suite of biomarkers of genotoxicity that would be applicable in toxicity testing are listed in Table 7.1. Several salient features of this suite are 
Table 7.1. Biomarters of genotoricity/carcinogenicity

\begin{tabular}{lccccc}
\hline Blomarker & $\begin{array}{c}\text { Blological } \\
\text { response }\end{array}$ & $\begin{array}{c}\text { Level of biologlcal } \\
\text { organlzatlon }\end{array}$ & $\begin{array}{c}\text { Temporal } \\
\text { occurrence }\end{array}$ & $\begin{array}{c}\text { Retectlon } \\
\text { level }\end{array}$ & Limitations \\
\hline DNA damage & Adducts & Molecular & Early & Low & Repair/analysis \\
DNA damage & Strand breaks & Molecular & Early & Moderate & Repalr \\
DNA damage & Repair & Molecular & Early & Low & Analysis \\
Mixed function oxidase & Enzyme inductlon & Molecular & Early & Moderate & Species variabillty \\
DNA damage & Minor nucleoside & Blochemical & Early/middle & High & High \\
Chromosomal aberrations & Abnormal DNA & Subcellular & Middle/late & High
\end{tabular}

Temporal occurrence subsequent to exposure: early-hours to days; middle-days to weeks/months; late-weeks/months to years.

${ }^{b}$ Constraints on blomarker application: (1) detection level-anticipated probability of detecting biomarker and (2) limitation-factor(s) contributing to detection or affecting use of biomarker. 
1. Biomarkers employ methodologies that detect events that are points on a continuum far removed from the long-term effect (see Fig. 1.1).

2. Each biomarker detects a unique type of DNA damage (e.g, adduct, strand break, or hypomethylation) or the consequence of that damage (c.g., DNA repair or chromosomal aberrations). Blomarkers of detoxication are included because of the intimate relationship that exists between xenobiotic metabolism and DNA damage.

It is obvious that a different set of biomarkers could be chosen that constitutes a suite suitable for evaluating other endpoints such as reproductive fallure or loss of immune competence. Selection can be tailored to meet the needs of the situation. 


\section{LONG-TERM STRATEGY FOR DEVELOPING AND IMPLEMENTING A BIOMARKER-BASED BIOMONITORING CAPABILITY}

There can be little doubt that measurement of biomarker responses in organisms from contaminated sites offers great promise for providing information that can contribute to monitoring programs designed for hazard identification, hazard assessment, or risk prediction. The challenge is to develop and implement a research program that will permit this promise to be fully realized. Current understanding and application of biomarkers justify their immediate implementation in an environmental monitoring program at a pilot scale. However, the full potential of this methodology will be realized only after a larger data base of field and laboratory studies can be accumulated and analyzed. It is the purpose of this section to present some thoughts on a research strategy that is needed to develop the data needed to validate biomarkers and provide the scientific understanding necessary to interpret biomarker responses of environmental species. What is proposed is an evolving monitoring program that focuses broadly on evaluation of contamination in an array of ecosystem types. The challenges and obstacles to be addressed in such a program include

1. The quantitative and qualitative relationships between chemical exposure, biomarker responses, and adverse effects must be established.

2. Responses caused by chemical exposure must be distinguishable from natural variability (ecological and physiological variables, species-specific differences, and individual variability) if biomarkers are to be useful in evaluating contamination.

3. The validity of extrapolating from biomarker responses measured in individual organisms to some higher-level (e.g., population or community) effect must be established.

4. The use of exposure biomarkers in animal surrogates to evaluate the potential for human exposure should be explored.

What is outlined here is an ambitious multiyear research and development program (Fig. 8.1). Ideally, such a program would be implemented as part of a long-term, interagency, interdisciplinary activity among agencies and users with any of the applications outlined in Sect. 4. The key needs are

1. acceptance of a general strategy (as is outlined in this document) to ensure that comparable data is collected in all studies (regardless of the specific objectives of each study) and

2. some formal or informal mechanism for communicating results among users and for "brainstorming" to provide ongoing focus and direction to laboratory and field studies. 


\subsection{STATUS OF CURRENT CAPABILITIES}

It is clear that no one biomarker is the panacea that can evaluate chemical exposure and effects. Advancing the potential application of biomarkers must, therefore, be a collaborative enterprise integrating the skills of a variety of specialists. Although a number of individual researchers have evaluated specific blomarkers in laboratory and field experiments, much of this research has been limited to either (1) laboratory exposures of animals to a limited number of well-described model contaminants or (2) measurements of a single biomarker response in field-collected animals. More recently, however, a handful of research groups, mostly at government agencies and national laboratories, have begun to evaluate the responses of a suite of biomarkers in animals from polluted environments. The results have been encouraging: biomarker responses have correlated with the perceived degree of contamination, and the relative ranking of sites on the basis of molecular and biochemical responses agrees well with community level measures of ecosystem integrity. However, the same blomarkers have not been used in all the studies, repeated monitoring at the same sites is rare, and large-scale field studies have been limited almost exclusively to marine or aquatic environments.

Nevertheless, core capabilities for measuring a fairly wide array of candidate biomarkers do exist at federal agencies, national laboratories, and universities, and sufficient experience exists for making rational choices about selection and sampling of animal species. The primary impediments to major progress in applying this approach to environmental monitoring is the lack of a unifying mandate and the need for stable long-term funding.

\subsection{PROPOSED IMPLEMENTATION OF A BIOMARKER MONITORING PROGRAM}

This plan consists of five tasks that lead from preliminary proof-of-principle demonstration of the use of biomarkers to indicate exposure to eventual linking of biomarker responses to individual and ecosystem effects. Although phases are presented in roughly chronological order of development, we recognize that many components of each phase are interdependent and that laboratory-based research (see Sect. 5.4) will be needed in all phases to improve understanding of the pharmacological/pharmacodynamic responses of biomarkers in various exposure scenarios. 

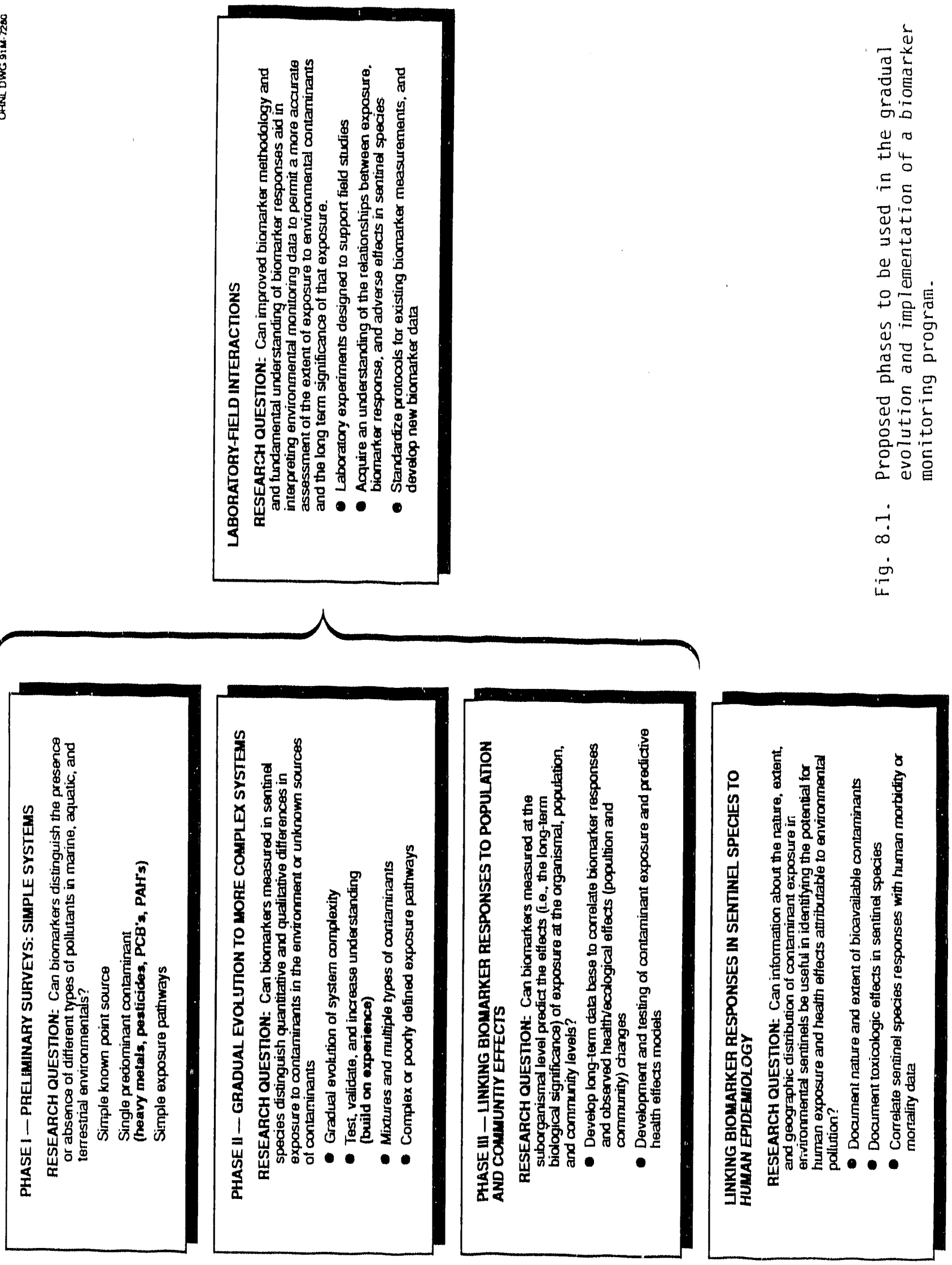


\subsubsection{Phase I: Start with Simple Systums}

Rescarch question-Can biomarkers distinguish the presence or absence of different types of pollutants in marine, aquatic, and terrestrial environments?

Research description-The adequacy of current understanding of, and approaches to, analysis of biomarkers in environmental species needs to be tested. Existing capabilities need to be consolidated and organized to test key questions at a very limited number of selected field sites. Results of this preliminary survey will demonstrate the strengths and weaknesses of current approaches, provide an initial screening of promising biomarkers, and establish QA/QC protocols, datu base logistics, and statistical analyses that can form the basis of a longuterm biomarker monitoring program.

Research objective-The objective of this research is to compare the qualitative pattern and quantitative responses of a suite of biomarkers in sentinel species from sites polluted with specific types of contaminants compared with the responses of organisms from pristine reference sites.

Approach—A core group of researchers from federal agencies, national laboratories, and universities will be identified to evaluate and compare a suite of the most promising biomarkers. Biomarkers will be selected by a panel active in biomarker research. A limited number of field sites, representing aquatic, marine, and terrestrial environments, will be identified for this initial study. Within each environment, selected field sites should present relatively simple exposure patterns, typified by predominance of a single type of contaminant (e.g., heavy metals, pesticides, or carcinogens such as PAHs), and simple exposure pathways. For each type of environment, one or more reference sites will be identified that is ecologically similar to the contaminated sites but is free of any known pollutant inputs. Sentinel species will be selected based on their habitat and trophic level to test the power of the biomarkers to identify the contribution of different routes of exposure.

Ideally, each site would be sampled 2 to 3 times a year for at least 2 years (although this may not be reasonable or possible for some applications). Each member of the core group could measure the response of the biomarkers for which they have expertise in animals collected at each of the different sites. The final product of this task will be a data base describing the responses of a suite of biomarkers at different levels of biological organization in the same species sampled at the same time 
from polluted and reference sites. Statistical analyses will evaluate (1) how well different biomarkers distinguished the presence or absence of known contaminants (i.e., Were they successful indicators of exposure?), (2) whether different types of contaminants zould be distinguished from the pattern of biomarker responses, and (3) how consistent the biomarkers were from season-to-season and year-to-year at the same sites (i.e., can they distinguish toxicant-related stress from natural ecological variability?).

\subsubsection{Phase II: Gradually Evolve to More Complex Systems}

Research quesiion-Can biomarkers measured in sentinel species distinguish quantitative and qualitative differences in exposure to contaminants in the environment?

Research description-This task builds on and extends the preliminary survey described in Phase I. That task sought to establish that the biomarkers could distinguish the presence or absence of exposure. This task begins the process of distinguishing relative degrees of exposure (e.g., differences in the extent of exposure at different sites within a polluted estuary or at increasing distances from a hazardous waste site). Likewise, while the first task selected sites characterized by a predominance of a single type of ontaminant and simple and clearly delineated exposure pathways, this task will begin to include sites with more complex mixtures of contaminants and greater complexities in exposure pathways and trophic dynamics. The first task sought to establish that biomarkers were useful in aquatic, terrestrial, and marine environments; this task will begin to explore the application of biomarkers in environments with wider geographic diversity (e.g., forests, prairies, and agroecosystems are diverse examples of terrestrial environments).

This task is not a single activity, but rather a gradual evolution from the simple proof-of-principle to a comprehensive biomarker-based biomonitoring capability. Experience in different types of environments and selection of sensitive and informative biomarkers derived in the first task will provide a foundation for expanding to more complex scenarios. Successful implementation of the biomarker approach to environmental monitoring requires this gradual building of capabilities and understanding. The same atiuibutes that make t! a biomarker approach a powerful tool for environmental monitoring also caution against its rapid anc indiscriminate application without the benefit of carefully accumulated experience. Biomarker responses are powerful because they 
integrate a wide array of environmental, toxicological, and ecological factors that control and modulate exposure to and effects of environmental contaminants. However, these same factors may also complicate interpretation of the significance of biomarker responses in ways that may not always be anticipated. Phased increases in the extent and complexity of monitoring scenarios will test and confirm previous understanding and gradually' expand that understanding until the biomarkers become a routine, well-characterized, and scientifically and legally defensible tool for monitoring and assessing environmental pollution.

Research objective-The objective of this research is to increase understanding of the qualitative pattern and quantitative response of a suite of biomarkers in sentinel species until a capability is developed to identify the extent of exposure and the nature of the contaminant to which the organisms are being exposed. The emphasis of this task is on developing a capability to assess exposure rather than to evaluate the adverse effect or long-term biological significance of that exposure.

Approach-Experiences gained from Phase I will be the starting point for this task. The suite of the most effective biomarkers selected from the first task will be applied to additional sampling locations in the same areas studied in that task. The number and complexity of field sites and contamination scenarios will be gradually increased to include more-complex suites of contaminants, more-complex exposure pathways, and greater geographic diversity. New biomarkers should be tested as field sampling increases, and their value compared with those already in use. Improved statistical analyses are needed to describe the pattern and geographic distribution of biomarker responses. With each increase in complexity, results need to be analyzed to evaluate:

1. how well the biomarkers distinguish quantitative differences in the extent of exposure;

2. whether the pattern of responses of the suite of biomarkers can distinguish the nature of the contaminants to which the organisms were exposed (e.g., Are metals or carcinogens the primary toxic agents to which the organisms are responding?);

3. how effectively the biomarkers in selected sentinel species distinguish the significance of different routes of exposure (e.g., air, water, soil, sediment, and/or food chain); and

4. whether the quantitative and qualitative responses of the biomarkers can distinguish toxicant exposure in the presence of natural environmental, ecological, and physiological variables. 


\subsubsection{Phase III: Linking Biomarker Responses to Community-Level Effects}

Research question-Can biomarkers measured at the suborganismal level predict the effects (i.e., the long-term biological significance) of exposure at the organismal, population, and community level?

Research description-Although estimating differences between or changes in patterns of exposure is clearly a critical objective of an environmental monitoring program, questions concerning the significance of that exposure to the long-term health and well-being of the organism or its ecosystem must ultimately be addressed. If there is some detectable level of exposure, is it "bad"? What level of exposure will lead to unacceptable or undesirable deterioration of the environment, measured, for example, in terms of the loss of commercially important species or in terms of some more subjective aesthetic criteria. Conversely, "how clean is clean?" Can we determine when pollutant inputs are low enough to cause no long-term adverse effects? This is a critical question in decisions about remediation of existing pollution or in permitting discharges of chemicals into the environment.

The effects of contaminant exposure are difficult to address unequivocally for two reasons:

1. There is often a long latent period between exposure and the measurable expression of an often irreversible adverse effect.

2. It is often very difficult to attribute effects observed in field studies to toxicant exposure because of biological variability among individual organisms and confounding influences of natural environmental and ecological factors such as food and habitat availability.

Implementation of a long-term monitoring program that measures biological markers at different ievels of biological organization (see Fig. 1.2) offers the potential for acquiring the information necessary to link sensitive and specific responses to toxicant exposure (measured at the molecular, biochemical, or physiological level) with long-term responses at the organismic, population, and community levels. Many of the suborganismal biomarkers respond quickly to changes in toxicant exposure and can be more clearly linked to specific actions of pollutants. The data base developed in a monitoring program will demonstrate the relationships between quantitative and qualitative responses in these biomarkers and long-term adverse effects at the organism level (e.g., increased susceptibility to disease or reduced fecundity) and at the population and community levels (changes in the structure of function of the system). 
Although this is a complex task and needs to include research components involving experimental validation and ecological modeling, the dividends are very significant in terms of environmental protection and cost-savings.

1. The environmental significance of changes in pollutant inputs to an ecosystem can be rapidly assessed to allow intervention before irreversible damage occurs or to guide cost-effective remediation or input reductions.

2. Chronic bioassays can be devised whose endpoints are changes in rapidly responding biochemical or molecular biomarkers but which provide information on the long-term, population level consequences of exposure to the test chemicals.

3. The costs of the environmental monitoring program will be reduced because measurements of biomarkers at the higher levels of biological organization can be reduced or eliminated.

Research objective-The objective of this task is to establish and verify the relationships between exposure, changes in rapidly-responding suborganismal biomarkers, and long-term adverse effects at the population or community level. The successful completion of this objective will validate the use of molecular-, biochemical-, and physiological-level biomarkers as indicators of the health status of organisms and populations (see Fig. 1.2).

Approach-Phase III will develop a long-term data base on responses of biomarkers at many levels of biological organization in a diversity of environments affected to varying extents by an array of contaminants. These data need to be analyzed to establish correlations between suborganismal biomarker responses and population and community structure and function. These correlations will permit hypotheses, the formulation of which must then be tested in long-term microcosm or mesocosm experiments where exposure can be controlled. The time course of biomarker responses can be monitored and evaluated with respect to the ability of the biomarkers to forecast communitylevel responses. Relationships between molecular and biochemical markers and population-level effects may not be straightforward because of compensatory mechanisms that help regulate population dynamics in natural systems. Ecosystem models that incorporate complex trophic struciures and describe higher-level compensatory mechanisms need to be evaluated as possible ways of describing and predicting links between these different levels of organization and complexity. Because fecundity is a key parameter linking individual responses and ecosystem processes, particular attention should be directed toward evaluating the molecular, biochemical, or endocrinological factors regulating the 
reproductive capacity of an individual organism. These reproductive parameters, coupled with ecosystem models, may be useful as tools in ecological risk assessment.

\subsubsection{Laboratory-Field Interaction: Development, Standardization, and Validation of Key Biomarkers}

Research question-Can improved biomarker methodology and fundamental understanding of biomarker responses aid in interpreting environmental monitoring data to permit a more accurate assessment of the extent of exposure to environmental contaminants and the long-term significance of that exposure.

Research description-This task provides a framework for most of the fundamental laboratory research required to develop and hone the most powerful and informative biomarkers and to acquire the fundamental understanding necessary to interpret the significance of and interactions among biomarker responses. The principal thrust of this task will be directed toward laboratory research in which environmental species are used as sentinels. However, research focused on human health or basic biomedical studies can also contribute to advancing the objectives of this task. For example, advances in development of sophisticated biomarker methodology for estimating exposure of humans in the workplace, including development of monoclonal antibodies or DNA probes for adducts or advanced analytical or clinical technologies that increase the sensitivity, selectivity, or speed of biomarker measurements, can contribute significantly to the application of biomarkers in an environmental monitoring program. Likewise, basic biomedical research that increases understanding of toxicant action at a molecular and cellular level will enhance capabilities to interpret biomarker responses because most of the biochemical processes in environmental species are comparable to those of laboratory animals used in biomedical research. Although research focused on human health issues would not be part of the monitoring program, limited funds may permit biomedical researchers to extend their studies to make results relevant to understanding biomarker responses in animal sentinels.

Research objective-The objective of this research is to standardize protocols for existing biomarker measurements, develop and modify new biomarkers as needed, and acquire the fundamental 
understanding of the relationships between exposure, hirarker responses, and adverse effects in sentinel species at the cellular, organismic, or population level.

Approach-Laboratory exposures with single contaminants or rationalized mixtures of contaminants are needed to establish dose-dependent relationships between exposure, biomarker responses, and toxic effects in sentinel species (see Sect. 5.4). Promising biomarker methods developed for certain species (including humans) may need to be modified to extend their application to a wider range of environmental species, including plants. Biomarkers that are selected for long-term monitoring prograxis noted to be optimized for individual species, and standardized protocols need to be tested by interlaboratory comparisons. The role of natural environmental, ecological, and physiological variables (such as seasonal changes in temperature, food availability, and reproductive status) on the magnitude and pattern of biomarker responses must be determined. The time course of biomarker responses will be an important variable in interpreting the extent and significance of exposure and must be evaluated in various sentinel species.

The task will involve an ongoing interaction with field monitoring programs and research described in other tasks. Difficulties in interpreting biomarker responses in the field will be resolved through laboratory research in this task. Valuable and informative biomarkers that require laborious (and expensive) procedures will be automated or otherwise improved to make them more cost-effective for a routine monitoring program. New insights into biochemical aspects of toxicology will be applied and tested in species used as environmental sentinels to improve understanding and interpretation of monitoring data.

\subsubsection{Phase IV: Linking Biomarker Responses in Sentinel Species to Human Epidemiology}

Research question-Can information about the nature, extent, and geographic distribution of contaminant exposure in environmental sentinels be useful in identifying the potential for human exposure and health effects attributable to environmental pollution?

Research description-The presence of toxic and carcinogenic pollutants in the environment raises obvious concerns about the potential for human health effects resulting from environmental exposures. However, it is very difficult, from an epidemiological perspective, to attribute any increase 
in health risk to environmental sources. Measurements of biomarkers in environmental sentinels may be useful in evaluating the human health effects associated with exposure to environmental contaminants. A health-effect evaluation has four fundamental phases:

1. documentation of the nature and extent of exposure,

2. definition of exposed and unexposed populations,

3. diagnosis of disease in exposed populations, and

4. establishing relationships between exposure and disease.

An environmental monitoring plan using biomarkers in environmental species can contribute directly to documenting the nature and extent of bioavailable contaminants, whereas the geographic pattern of animal sentinel responses can identify areas of human habitation that show evidence of bioavailable environmental contaminants. The animal sentinels provide a method for attributing risks to environmental sources of exposure because animals do not share human exposure from life style or workplace pollutants. Furthermore, observations on the toxicologic effects of realistic chronic exposure in animals in contaminated environments can contribute to understanding the relationship between exposure and disease in humans. Although this task may not be central motivation for an environmental monitoring program, the potential benefits to human health assessment should not be overlooked.

Research objective-The objective of this research is to determine whether there are correlations between patterns in the extent, nature, and geographic distribution of biomarker responses in sentinel species and patterns of epidemiological evidence of increased risk to human health.

Approach-As Phase III is implemented and expanded, information will become available on biomarker responses in sentinel species over relatively large geographic regions, including areas of human habitation. The pattern of biomarker responses (quantitative and qualitative) can be represented on a geographic map in which isopleths define areas of higher or lower levels of bioavailable contaminants. Superimposition of such a map of sentinel responses on an analogous map of human morbidity or mortality data may provide indications of correlations between environmental contaminant levels (biomarker responses of sentinels) and risks of human health effects. 


\subsection{SCHEDULE FOR IMPLEMENTATION OF BIOMARKER MONITORING PROGRAM}

The environmental monitoring program that emerges from this proposed research plan will be the final evolution of the field monitoring in Phase II, illuminated by laboratory research and development described in Phase III. Our capabilities to interpret the data base from this program will continue to grow as field experience and experimental validation accumulate over time. In terms of budget and effort, three phases are anticipated:

1. Initial Phase-Starting with initial workshops and planning sessions, Task I will begin to be implemented. Sampling and analyses at the selected field sites should be phased in slowly to gain experience in and overcome initial problems with logistics, data base management, QA/QC protocols, and other complications expected in multi-institutional collaborations. Budgets will increase in the second and third year as the full matrix of field sites, sentinel species, and suites of biomarkers for Task I are implemented and data begins to be analyzed.

2. Lecrning Phase-Most of the activities described in Tasks II through IV will occur during this phase of implementation. Budgets and manpower commitments will rise substantially and peak in the latter half of this phase, which is expected to last for several years. Field sampling (Task III) and laboratory research and development (Task II) will be heaviest early in this phase and will level off as experience is gained and understanding is achieved. Tasks IV and V require an accumulation of field data, and activity in these components will begin to rise at about the middle of this learning phase.

3. Routine Monitoring Phase-This is the mature phase of biomarker-based biomonitoring. Most of the field monitoring protocols will have been established, biomarkers and sentinel species selected, and protocols for data analysis and interpretation standardized. Testing of new biomarkers, laboratory research to understand biomarker responses, improvements in methods for data analysis and interpretation, and other components from the Phase 2 (Learning Phase) will always continue at some level so that the power and utility of the monitoring program will continue to grow. Budgets and manpower requirements will decrease gradually during this phase and level off as monitoring and interpretation become more routine and as new technologies reduce the cost of biomarker measurements. 


\section{CONCLUSIONS}

Biomarkers measured in environmental spectes have been demonstrated to be useful indicators of exposure to and effects of contaminants in research studies and in a limited number of fleld evaluations. Because of the interdisciplinary nature of biomarker studies and the need for integration of numerous research specialties, long-term progress will be accelerated by general agreement of a common research strategy. The proposal described here is intended as a "strawman" to serve as a basis for continuing dialogue toward defining an acceptable umbrella strategy. Planning and implementation of such a strategy would be facilitated if it were part of a larger interagency mandate. However, all that is really needed is for the community of researchers involved in biomarker research, in the United States and abroad, to decide on their common strategy and to keep in communication with each other to integrate results and to help one another interpret their observations. This could be accomplished ty informal gatherings at regularly attended scientific meetings, by organization of Gordon Conference-type meetings at regular intervals, or by more formal workshops funded by consortia of interested government agencies. We hope that this "strawman" will be reviewed, expanded, honed, and endorsed by international experts at a NATO Advanced Research Workshop in 1991. Progress in development and validation of biomarker-based biomonitoring will be improved by adoption of such an internationally accepted strategy. 


\section{REFERENCES}

Adams, S. M., J. J. Beauchamp, and C. A. Burtis, 1988. A multivariate approach for evaluating responses of fish to chronic pollution stress. Mar, Environ. Res, 24:223-26.

Adams, S. M., K. L. Shepard, M. S. Greeley, B. D. Jimenez, M. G. Ryon, L. R. Shugart, and J. F. McCarthy. 1989. The use of bioindicators for assessing the effects of pollutant stress on fish. Mar. Environ. Res. 28:459-64.

Adams, S. M., L. R. Shugart, G. R. Southworth, and D. E. Hinton. 1990. Application of bloindicators in assessing the health of fish populations experiencing contaminant stress. pp. 33353. IN J. F. McCarthy and L. R. Shugart (eds.), Biological Markers of Environmental Contamination. Lewis Publishers, Inc., Boca Raton, Fla.

Carnes, S. A., and A. P. Watson. 1989. Disposing of the U.S. Chemical Weapons Stockpile. J. Am. Med. Assoc. 262: 653-59.

Christian, J. J. 1983. Love canals unhealthy voles. Nat. Hist. 10:8-16.

Depledge, M. 1989. The rational basis for detection of the early effects of marine pollutants using physiological indicators. AMBIO 18:301-02.

Farber, E. 1987. Possible etiologic mechanisms in chemical carcinogenesis. Environ. Health Perspect. 75:65-70.

Harvey, R. G. 1982. Polycyclic hydrocarbons and cancer. Am. Sci. 70:386-93.

Hawkins, W. E., R. M. Overstreet, and W. W. Walker. 1988. Carcinogenicity tests with small fish species. Aquat. Toxicol. 11:113-28.

Henderson, R. F., W. E. Bechtold, J. Bond, and J. D. Sun. 1989. The use of biological markers in toxicology. Toxicology 20:65-82.

Huggett, R., R. A. Kimerle, P. M. Mehrle, and H. L. Bergman, eds. 1991. Biomarkers: Biochemical, Physiological, and Histological Markers of Anthropogenic Stress. Lewis Publishers, Inc., Boca Raton, Fla.

Hulka, B. S., and T. Wilcosky. 1988. Biological markers in epidemiologic research. Arch. Environ. Health. 43:83-89.

Johnson, Alan. 1988. State space displacement analysis of the response of aquatic ecosystems to phenolic toxicants. Ph.D. Dissertation, The University of Tennessee, Knoxville, Tenn.

Jones, P. A. 1987. Role of DNA methylation in regulating gene expression, differentiation, and carcinogenesis, pp. 17-30. IN J. C. Barrett (ed.) Mechanisms of Environmental Carcinogenesis, Vol.1, CRC Press, Boco Raton, Fla. 
Jimenez, B. D., A. Oikari, S. M. Adams, D. E. Hinton, and J. F. McCarthy, 1990. Hepatic enzymes as biomarkers: interpreting the effects of environmental physiological and toxicological varlables. pp. 123-42. In J. F, McCarthy and L. R. Shugart (eds.), Blological Markers of Environmental Contamination. Lewis Publishers, Inc, Boca Raton, Fla.

Loar, J. M., S. M. Adams, H. L. Boston, B. D. Jimenez, J. F. McCarthy, J. G. Smith, G. R. Southworth, and A. J. Stewart. 1988. First annual report on the Y-12 plant biological monitoring and abatement program. ORNL/TM-10265. Oak Ridge National Laboratory, Oak Ridge, Tenn.

Martin, M. H., and P. J. Coughtrey. 1982. Blological Monitoring of Heavy Metal Pollution. Applied Science Publishers, London.

McCarthy, J. F., D. N. Jacobson, L. R. Shugart, and B. D. Jimenez. 1989. Pre-exposure to 3-methylcholanthrene increases benzo(a)pyrene adducts on DNA of bluegill sunfish. Mar. Environ. Res. 28:323-28.

McCarthy, J. F., and L. R. Shugart, eds. 1990. Blomarkers of Environmental Contamination. Lewis Publishers, Inc., Boca Raton, Fla.

NRC (Nuclear Regulatory Commission). 1987. Blological markers in environmental health research. Committee on Biological Markers of the National Research Council. National Academy of Sciences. Environ. Health Perspect. 74:3-9.

NRC (Nuclear Regulatory Commission). 1989. Biologic Markers in Reproductive Toxicology. National Academy Press, Washington, D.C.

Shugart, L. R. 1990. Biological Monitoring: Testing for Genotoxicity. pp. 205-16. IN J. F. McCarthy and L. R. Shugart, eds., Biomarkers of Environmental Contamination, Lewis Publishers, Inc., Boca Raton, Fla.

Shugart, L. R., S. M. Adams, B. D. Jimenez, S. S. Talmage, and J. F. McCarthy. 1989. Biological markers to study exposure in animals and bioavailability of environmental contaminants. pp. 8697. IN R. G. M. Wang, C. A. Franklin, R. C. Honeycutt, and J. C. Reinert, eds., Biological Monitoring for Pesticide Exposure: Measurement, Estimation, and Risk Reduction, ACS Symposium Series 382, Chap. 7, American Chemical Society, Washington, D. C.

Suter, G. W. 1990. Use of biomarkers in ecological risk assessment. pp. 419-426. IN Biological Markers of Environmental Contamination. J. F. McCarthy and L. R. Shugart, eds, Lewis Publishers, Inc., Boca Raton, Fla.

Talmage, S. S. 1989. Comprehensive evaluation of several small mammal species as monitors of heavy metals, radionuclides, and selected organic compounds in the environment. Ph.D. Dissertation, University of Tennessee, Knoxville, Tenn.

Thoman, R. V. 1981. Equilibrium model of fate of microcontaminants in diverse aquatic food chains. Can. J. Fish. Aquat. Sci. 38:280-96. 
Weldemeyer, G. A., D. J. McLeoy, and C. P. Goodyear. 1984. Assessing the tolerance of fish populations to environmental stress: The problem and methods of monitoring, pp. 163-95. IN V. W. Cairns, P. V. Hodson, and J. O. Nriagu (eds.), Contaminant Effects on Fisheries. Willey, New York. 


\section{INTERNAL DISTRIBUTION}

1. L. D. Bates

2. J. H. Cushman

3. M. P. Farrell

4. D. E. Fowler

5. C. W. Gehrs

6. R. S. Halbrook

7. S. G. Hildebrand

8. J. F. McCarthy

9. D. E. Reichle

10. D. S. Shriner
11. L. R. Shugart

12. S. H. Stow

13. R. I. Van Hook

14. Central Research Library

15-29.ESD Library

30-31.Laboratory Records Department

32. Laboratory Records, ORNL-RC

33. ORNL Patent Section

34. ORNL Y-12 Technical Library

\section{EXTERNAL DISTRIBUTION}

35. A. P. Dufour, Environmental Monitoring Systems Laboratory, U.S. Environmental Protection Agency, Cincinnati, OH 45268

36. J. F. Franklin, Bloedel Professor of Ecosystem Analysis, College of Forest Resources, University of Washington, Anderson Hall (AR-10), Seattle, WA 98195

37. G. M. Hornberger, Professor, Department of Environmental Sciences, University of Virginia, Charlottesville, VA 11903

38. G. Y. Jordy, Director, Office of Program Analysis, Office of Energy Research, ER-30, G-226, U.S. Department of Energy, Washington, DC 20545

41. R. H. Olsen, Vice President for Research, University of Michigan, 6643 Medical Science Building II, Ann Arbor, MI 48109-0620

42. A. Patrinos, Acting Director, Environmental Sciences Division, Office of Health and Environmental Research, ER-74, U.S. Department of Energy, Washington, DC 20585

42. F. J. Wobber, Environmental Sciences Division, Office of Health and Environmental Research, Office of Energy Research, ER-74, U.S. Department of Energy, Washington, DC 20585

43. Office of Assistant Manager for Energy Research and Development, Oak Ridge Operations, P. O. Box 2001, L.S. Department of Energy, Oak Ridge, TN 37831-8600

44. Office of Scientific and Techrical Information, P. O. Box 62, Oak Ridge, TN 37831 

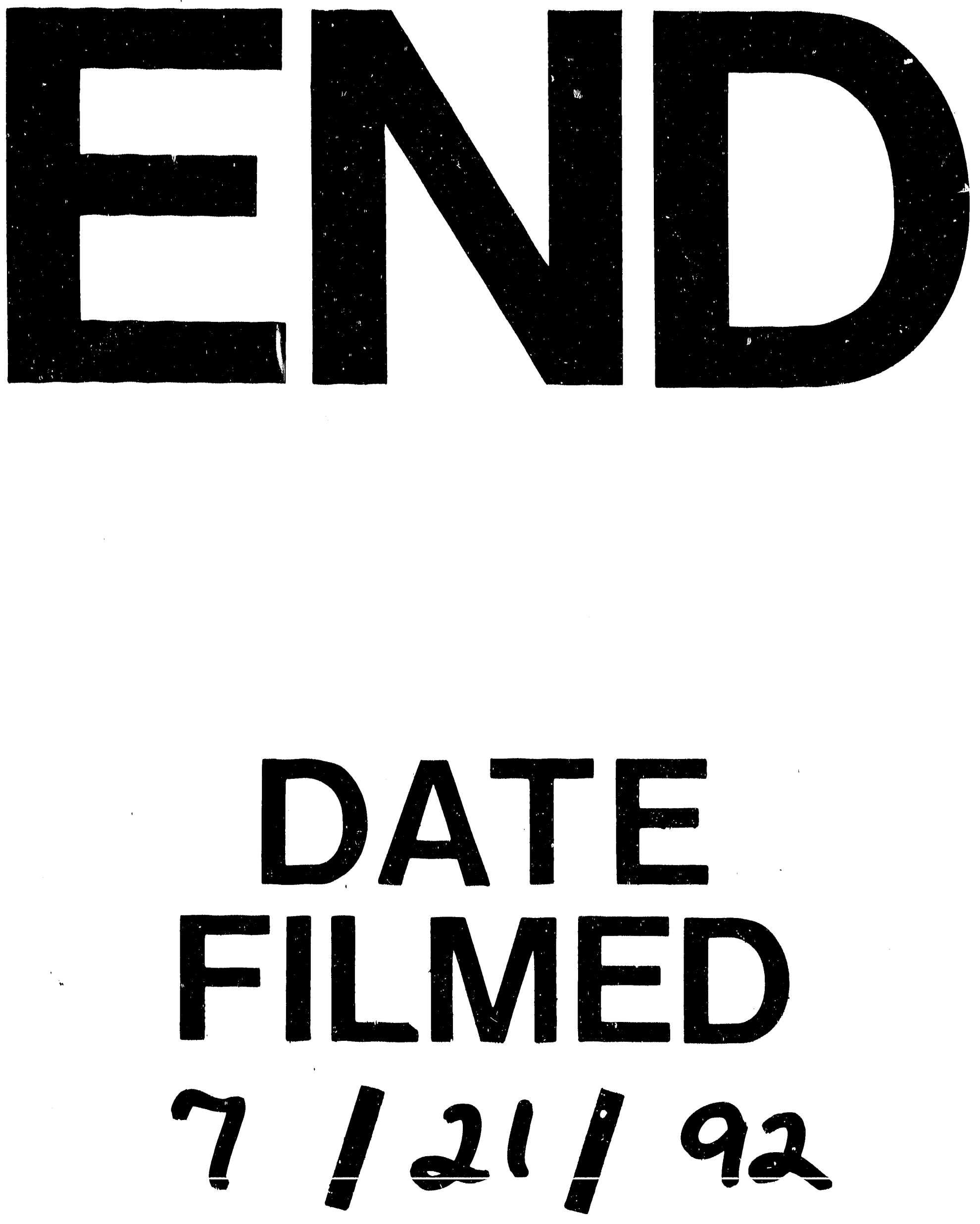

I 